\title{
A MODEL FOR TWO COUPLED TURBULENT FLUIDS PART II: NUMERICAL ANALYSIS OF A SPECTRAL DISCRETIZATION*
}

\author{
C. BERNARDI ${ }^{\dagger}$, T. CHACÓN REBOLLO ${ }^{\ddagger}$, R. LEWANDOWSKI ${ }^{\S}$, AND F. MURAT ${ }^{\dagger}$
}

\begin{abstract}
We consider a system of equations that models the stationary flow of two immiscible turbulent fluids on adjacent subdomains. The equations are coupled by nonlinear boundary conditions on the interface which is here a fixed given surface. We propose a spectral discretization of this problem and perform its numerical analysis. The convergence of the method is proven in the two-dimensional case, together with optimal error estimates.
\end{abstract}

Key words. turbulent fluids, numerical analysis, spectral discretization

AMS subject classifications. 65N35, 76D99

PII. S0036142901385829

1. Introduction. In this paper, we are interested in the numerical analysis of the spectral discretization of a model for two stationary turbulent fluids coupled by boundary conditions on the interface:

$$
\begin{cases}-\operatorname{div}\left(\alpha_{i}\left(k_{i}\right) \nabla \boldsymbol{u}_{i}\right)+\operatorname{grad} p_{i}=\boldsymbol{f}_{i} & \text { in } \Omega_{i}, 1 \leq i \leq 2, \\ \operatorname{div} \boldsymbol{u}_{i}=0 & \text { in } \Omega_{i}, 1 \leq i \leq 2, \\ -\operatorname{div}\left(\gamma_{i}\left(k_{i}\right) \nabla k_{i}\right)=\alpha_{i}\left(k_{i}\right)\left|\nabla \boldsymbol{u}_{i}\right|^{2} & \text { in } \Omega_{i}, 1 \leq i \leq 2, \\ \boldsymbol{u}_{i}=\mathbf{0} & \text { on } \Gamma_{i}, 1 \leq i \leq 2, \\ k_{i}=0 & \text { on } \Gamma_{i}, 1 \leq i \leq 2, \\ \alpha_{i}\left(k_{i}\right) \partial_{n_{i}} \boldsymbol{u}_{i}-p_{i} \boldsymbol{n}_{i}+\left(\boldsymbol{u}_{i}-\boldsymbol{u}_{j}\right)\left|\boldsymbol{u}_{i}-\boldsymbol{u}_{j}\right|=\mathbf{0} & \text { on } \Gamma, 1 \leq i \neq j \leq 2, \\ k_{i}=\left|\boldsymbol{u}_{1}-\boldsymbol{u}_{2}\right|^{2} & \text { on } \Gamma, 1 \leq i \leq 2,\end{cases}
$$

where each triple $\left(\boldsymbol{u}_{i}, k_{i}, p_{i}\right)$ is defined in the domain $\Omega_{i}, 1 \leq i \leq 2$. The vector field $\boldsymbol{u}_{i}$ represents the velocity of a turbulent fluid in $\Omega_{i}, p_{i}$ represents its pressure, and $k_{i}$ represents its turbulent kinetic energy (TKE in what follows). The domains $\Omega_{i}$ are two- or three-dimensional bounded open sets with common interface $\Gamma$, while each $\Gamma_{i}$ stands for $\partial \Omega_{i} \backslash \Gamma$.

${ }^{*}$ Received by the editors March 2, 2001; accepted for publication (in revised form) June 28, 2002; published electronically February 6, 2003.

http://www.siam.org/journals/sinum/40-6/38582.html

${ }^{\dagger}$ Laboratoire Jacques-Louis Lions (U.M.R. 7598), C.N.R.S. \& Université Pierre et Marie Curie, boîte 187, 4 place Jussieu, 75252 Paris Cedex 05, France (bernardi@ann.jussieu.fr, murat@ann.jussieu.fr). The research of the fourth author was partially supported by Iberdrola Visiting Professors Programme.

${ }^{\ddagger}$ Departamento de Ecuaciones Diferenciales y Análisis Numerico, Universidad de Sevilla, Tarfia s/n, 41012 Sevilla, Spain (chacon@numer.us.es). The research of this author was partially supported by Spanish Government grant REN2000-1168-C02-01.

§IRMAR (U.M.R. 6625), Université de Rennes 1, Campus de Beaulieu, 35042 Rennes Cedex 03, France (lewandow@maths.univ-rennes1.fr). 
System (1.1) is motivated by the coupling of two turbulent fluids $F_{i}, i=1$ and 2 , which appears in the framework ocean/atmosphere or in the case of two layers of a stratified fluid (see, e.g., [16, Chaps. 1 and 3] or [18]). Note that, in these situations, the operator $-\operatorname{div}\left(\alpha_{i}\left(k_{i}\right) \nabla \cdot\right)$ in (1.1) should be replaced by a different one, derived from the deformation rate tensor [11, sect. 2]. However, this change leads to more technical proofs, involving additional Korn-type inequalities, and we prefer to avoid it for simplicity of the presentation. These fluids $F_{i}$ are coupled through the interface condition on their common boundary $\Gamma$, which is supposed to be fixed. Indeed, we assume that the so-called "rigid lid hypothesis" holds, which is standard in geophysics and oceanography. According to this assumption, $\Gamma$ is a fixed mean interface and in fact the values of $\boldsymbol{u}_{i}, p_{i}$, and $k_{i}$ on $\Gamma$ are mean values of the velocity, pressure, and TKE. This law characterizes mean momentum exchanges between the fluids (see [16, Chap. 1] and [1]), and it is derived in a rather different way from standard wall laws [21] (but the mathematical formulation is rather similar): the turbulent mixed layer of the two turbulent fluids is modelled by the sixth and seventh lines in (1.1) which summarize the information related to a realistic interface ocean/atmosphere (see, e.g., [16, sect. 1.4] for more details about this model). Slightly more realistic models, obtained, for instance, by adding the convection term $\boldsymbol{u}_{i} \cdot \nabla \boldsymbol{u}_{i}$ in the first line of problem (1.1) and/or the dissipative term $-\frac{1}{L} k_{i}^{\frac{3}{2}}$ (where $L$ represents the mixing length) in the right-hand side of the third line of this problem, can also be considered. Since their analysis relies on exactly the same arguments as for problem (1.1), we skip these further terms for brevity.

The analysis of problem (1.1) is performed in [3] for two- or three-dimensional domains $\Omega_{i}$ which are either convex or of class C ${ }^{1,1}$. In that paper, an equivalent variational formulation of problem (1.1) is written, where the equations for the TKE are taken in the transposition sense (see [23] and [17, Chap. 2, sect. 6] for the definition of a solution by tranposition). Indeed, due to the lack of regularity of the right-hand side in the third line of (1.1) which belongs only to $L^{1}\left(\Omega_{i}\right)$, a standard formulation cannot be used here. However, the present formulation by transposition allows one to derive a priori estimates. Next the existence of a solution is proved. The uniqueness of smooth solutions is also established under some rather restrictive assumptions on the parameters and the data, and some regularity properties of the solutions are derived when the domains $\Omega_{i}$ are two-dimensional rectangles. Note, moreover, that the transposition formulation of the equations on the TKE is equivalent to the standard variational one when the solution is sufficiently smooth. We also refer to [2] for a slightly different proof of the existence result.

In the present paper, we are interested in the spectral discretization of problem (1.1), which relies on the approximation by high-degree polynomials. For simplicity, we consider only the key geometry where the domains are rectangles or rectangular parallelepipeds. However, in order to take into account the possible anisotropy of the flows which can be induced by the large aspect ratios of the domains, we use different degrees of polynomials with respect to the horizontal and vertical variables. We propose a discrete problem which, as usual for spectral methods [7, Chap. III], relies on the variational formulation of the equations for the velocity, the pressure, and also the TKE: it combines a conforming approximation in these spaces of polynomials with the use of numerical integration relying on tensorized Gauss-Lobatto formulas.

As standard for nonlinear systems, the numerical analysis of the discrete problem is performed via the discrete implicit function theorem of Brezzi, Rappaz, and Raviart [10]. As for the continuous problem, the main difficulty is due to the lack of regularity of the right-hand sides in the discrete TKE equations, and, as far as we 
know, the numerical analysis of problems with data in $L^{1}$ has been performed only in a few works (see [14], [13], and [12]). Thanks to the Brezzi-Rappaz-Raviart theory, in the two-dimensional case, we derive the existence of a solution of the discrete problem in a neighborhood of a nonsingular exact solution under some reasonable assumptions on its regularity. We also prove the convergence of the method, together with optimal error estimates. The same properties hold in the three-dimensional case; however, we think that the assumptions that are needed to prove them are no longer reasonable. A different analysis, leading to weaker convergence results, is under consideration.

To conclude, we propose an algorithm for solving the discrete problem. Its convergence is currently checked via numerical experiments and is likely at least for small variations of the functions $\alpha_{i}$ and $\gamma_{i}$.

The numerical analysis of the finite element discretization of system (1.1) is under consideration, and its convergence seems to be likely in the two- and three-dimensional cases under realistic assumptions.

An outline of the paper is as follows.

- In section 2 , we recall from [3] the variational formulation and the main properties of problem (1.1). We also write a different formulation in view of the discretization.

- In section 3, we describe the choice of the approximation spaces and the discrete problem. We also write a different and equivalent formulation of this problem, which is needed for its analysis.

- Section 4 is devoted to the numerical analysis of the discrete linear Laplace and Stokes problems with variable coefficients that are involved in the discretization.

- In section 5, we perform the numerical analysis of the coupled system. We prove the existence of a solution and derive error estimates.

- In section 6, we propose some conclusions and present a numerical algorithm for solving the discrete problem in the two-dimensional case.

2. Main properties of the continuous problem. In what follows, $\Omega_{1}$ and $\Omega_{2}$ stand for disjoint bounded domains in $\mathbb{R}^{d}, d=2$ or 3 , which are either convex or of class $C^{1,1}$. The generic point in $\mathbb{R}^{2}$ (resp., in $\mathbb{R}^{3}$ ) is denoted by $\boldsymbol{x}=(x, z)$ (resp., $\boldsymbol{x}=(x, y, z))$. We assume for simplicity that the interface $\Gamma=\partial \Omega_{1} \cap \partial \Omega_{2}$ coincides with the intersection of both $\bar{\Omega}_{1}$ and $\bar{\Omega}_{2}$ with the hyperplane $z=0$, while $\Omega_{1}$ and $\Omega_{2}$ are contained in the half-spaces $z>0$ and $z<0$, respectively. We denote by $\Gamma_{i}$ the part of the boundary $\partial \Omega_{i} \backslash \Gamma$. It must be noted that, in a number of practical situations, the vertical heights of the $\Omega_{i}$ are much smaller than their horizontal diameters.

Throughout the paper, we assume that the functions $\alpha_{i}$ and $\gamma_{i}, 1 \leq i \leq 2$, are continuous and bounded on $\mathbb{R}$, and are continuously differentiable with bounded derivatives. Moreover, we assume that there exists a positive constant $\nu$ such that, for $1 \leq i \leq 2$,

$$
\forall k \in \mathbb{R}, \quad \alpha_{i}(k) \geq \nu \quad \text { and } \quad \gamma_{i}(k) \geq \nu .
$$

We now write a variational formulation of problem (1.1). Next we recall its properties. Finally, we write another formulation of it that relies on the introduction of the Stokes and Laplace operators.

The variational formulation. Throughout the paper, we use the spaces $L^{p}\left(\Omega_{i}\right)$, $1 \leq p \leq \infty$, and the Sobolev spaces $H^{s}\left(\Omega_{i}\right)$ and $H_{0}^{s}\left(\Omega_{i}\right)$ for any real number $s$, provided with the standard norm $\|\cdot\|_{H^{s}\left(\Omega_{i}\right)}$ and seminorm $|\cdot|_{H^{s}\left(\Omega_{i}\right)}$, together with their analogues on $\Gamma$. We also need the special space $H_{00}^{\frac{1}{2}}(\Gamma)$, defined, e.g., in [17, Chap. 1, Thm. 11.7]. 
For $1 \leq i \leq 2$, we introduce the spaces

$$
X_{i}=\left\{\boldsymbol{v}_{i} \in H^{1}\left(\Omega_{i}\right)^{d} ; \boldsymbol{v}_{i}=\mathbf{0} \text { on } \Gamma_{i}\right\} .
$$

For reasons explained in [3, sect. 2], we also define the functions $G_{i}, 1 \leq i \leq 2$, by

$$
G_{i}(k)=\int_{0}^{k} \gamma_{i}(\kappa) d \kappa
$$

Problem (1.1) can be written (see [2] and [3]) as the following variational system of two coupled problems:

Find $\left(\boldsymbol{u}_{i}, p_{i}\right)$ in $X_{i} \times L^{2}\left(\Omega_{i}\right), 1 \leq i \leq 2$, such that, for $1 \leq i \neq j \leq 2$,

$$
\begin{aligned}
\forall \boldsymbol{v}_{i} \in X_{i}, \quad \int_{\Omega_{i}} \alpha_{i}\left(k_{i}\right) \nabla \boldsymbol{u}_{i}: & \nabla \boldsymbol{v}_{i} d \boldsymbol{x}-\int_{\Omega_{i}} p_{i}\left(\operatorname{div} \boldsymbol{v}_{i}\right) d \boldsymbol{x} \\
& +\int_{\Gamma}\left|\boldsymbol{u}_{i}-\boldsymbol{u}_{j}\right|\left(\boldsymbol{u}_{i}-\boldsymbol{u}_{j}\right) \cdot \boldsymbol{v}_{i} d \tau=\int_{\Omega_{i}} \boldsymbol{f}_{i} \cdot \boldsymbol{v}_{i} d \boldsymbol{x},
\end{aligned}
$$

$\forall q_{i} \in L^{2}\left(\Omega_{i}\right), \quad-\int_{\Omega_{i}} q_{i}\left(\operatorname{div} \boldsymbol{u}_{i}\right) d \boldsymbol{x}=0 ;$

Find $k_{i}$ in $L^{2}\left(\Omega_{i}\right), 1 \leq i \leq 2$, such that, for $1 \leq i \leq 2$,

$$
\begin{aligned}
& \forall \varphi_{i} \in H^{2}\left(\Omega_{i}\right) \cap H_{0}^{1}\left(\Omega_{i}\right), \\
& \quad-\int_{\Omega_{i}} G_{i}\left(k_{i}\right) \Delta \varphi_{i} d \boldsymbol{x}=-\int_{\Gamma} G_{i}\left(\left|\boldsymbol{u}_{1}-\boldsymbol{u}_{2}\right|^{2}\right) \partial_{n_{i}} \varphi_{i} d \tau \\
& +\int_{\Omega_{i}} \alpha_{i}\left(k_{i}\right)\left|\nabla \boldsymbol{u}_{i}\right|^{2} \varphi_{i} d \boldsymbol{x} .
\end{aligned}
$$

Note that the equations for the velocities and the pressure are of standard variational type and involve the bilinear forms, for $1 \leq i \leq 2$,

$$
a_{i}\left(t_{i} ; \boldsymbol{u}_{i}, \boldsymbol{v}_{i}\right)=\int_{\Omega_{i}} \alpha_{i}\left(t_{i}\right) \nabla \boldsymbol{u}_{i}: \nabla \boldsymbol{v}_{i} d \boldsymbol{x}, \quad b_{i}\left(\boldsymbol{v}_{i}, q_{i}\right)=-\int_{\Omega_{i}} q_{i}\left(\operatorname{div} \boldsymbol{v}_{i}\right) d \boldsymbol{x}
$$

However, the equation on the TKE is formulated in the transposition sense of Stampacchia [23] and of Lions and Magenes [17, Chap. 2, sect. 6].

As standard for the Stokes problem, we consider the kernel

$$
V_{i}=\left\{\boldsymbol{v}_{i} \in X_{i} ; \operatorname{div} \boldsymbol{v}_{i}=0 \text { in } \Omega_{i}\right\},
$$

and we observe that, for each solution $\left(\boldsymbol{u}_{i}, p_{i}\right)$ of problem (2.4), the velocity $\boldsymbol{u}_{i}$ is a solution of the following problem:

Find $\boldsymbol{u}_{i}$ in $V_{i}, 1 \leq i \leq 2$, such that, for $1 \leq i \neq j \leq 2$,

$$
\begin{aligned}
\forall \boldsymbol{v}_{i} \in V_{i}, \quad \int_{\Omega_{i}} \alpha_{i}\left(k_{i}\right) \nabla \boldsymbol{u}_{i} & : \nabla \boldsymbol{v}_{i} d \boldsymbol{x} \\
& +\int_{\Gamma}\left|\boldsymbol{u}_{i}-\boldsymbol{u}_{j}\right|\left(\boldsymbol{u}_{i}-\boldsymbol{u}_{j}\right) \cdot \boldsymbol{v}_{i} d \tau=\int_{\Omega_{i}} \boldsymbol{f}_{i} \cdot \boldsymbol{v}_{i} d \boldsymbol{x} .
\end{aligned}
$$

Conversely, we recall from [3, Lem. 3.1] that, for $1 \leq i \leq 2$, there exists a positive constant $\beta_{i}$ such that the following inf-sup condition holds:

$$
\forall q_{i} \in L^{2}\left(\Omega_{i}\right), \quad \sup _{\boldsymbol{v}_{i} \in X_{i}} \frac{b_{i}\left(\boldsymbol{v}_{i}, q_{i}\right)}{\left\|\boldsymbol{v}_{i}\right\|_{H^{1}\left(\Omega_{i}\right)^{d}}} \geq \beta_{i}\left\|q_{i}\right\|_{L^{2}\left(\Omega_{i}\right)} .
$$


This yields, for any solution $\boldsymbol{u}_{i}$ of problem (2.7), the existence of a unique function $p_{i}$ in $L^{2}\left(\Omega_{i}\right)$ such that the pair $\left(\boldsymbol{u}_{i}, p_{i}\right)$ is a solution of problem (2.4). So, for the next results, we work with the simpler system $(2.5)-(2.7)$.

Main properties. We first recall from [3, Lems. 3.3 and 4.2] the following a priori estimates: for any $\boldsymbol{f}_{i}$ in $L^{2}\left(\Omega_{i}\right)^{d}, 1 \leq i \leq 2$, every solution $\left(\boldsymbol{u}_{1}, \boldsymbol{u}_{2}\right)$ of problem (2.7) satisfies

$$
\left\|\boldsymbol{u}_{1}\right\|_{H^{1}\left(\Omega_{1}\right)^{d}}+\left\|\boldsymbol{u}_{2}\right\|_{H^{1}\left(\Omega_{2}\right)^{d}} \leq \frac{c}{\nu}\left(\left\|\boldsymbol{f}_{1}\right\|_{L^{2}\left(\Omega_{1}\right)^{d}}+\left\|\boldsymbol{f}_{2}\right\|_{L^{2}\left(\Omega_{2}\right)^{d}}\right)
$$

and, for any real number $s, 0 \leq s<\frac{1}{2}$, and for $1 \leq i \leq 2$, every solution $\ell_{i}$ of problem (2.5) satisfies

$$
\left\|\ell_{i}\right\|_{H^{s}\left(\Omega_{i}\right)} \leq c_{s}\left(\left\|\boldsymbol{u}_{1}\right\|_{H^{1}\left(\Omega_{1}\right)^{d}}^{2}+\left\|\boldsymbol{u}_{2}\right\|_{H^{1}\left(\Omega_{2}\right)^{d}}^{2}\right) .
$$

The constants $c$ and $c_{s}$ depend on the geometry of $\Omega$, on $\nu$, and on the maximal value of the $\alpha_{i}$ and $\gamma_{i}$; moreover, the constant $c_{s}$ depends on $s$.

Using these estimates, an existence result is proved in [3, Cor. 5.3]. We only state it.

THEOREM 2.1. For any $\boldsymbol{f}_{i}$ in $L^{2}\left(\Omega_{i}\right)^{d}, 1 \leq i \leq 2$, system (2.4)-(2.5) has a solution $\left(\tilde{U}_{1}, \tilde{U}_{2}\right)$ with each $\tilde{U}_{i}=\left(\boldsymbol{u}_{i}, p_{i}, k_{i}\right)$ in $X_{i} \times L^{2}\left(\Omega_{i}\right) \times L^{2}\left(\Omega_{i}\right)$. Moreover, each function $k_{i}, i=1$ and 2 , is nonnegative and belongs to $H^{s}\left(\Omega_{i}\right)$ for all $s<\frac{1}{2}$.

In contrast, the uniqueness result in [3] (see also [11] for a similar result) is rather disappointing. It states that, if system (2.4)-(2.5) admits a solution $\left(\boldsymbol{u}_{i}, p_{i}, k_{i}\right)_{1 \leq i \leq 2}$ such that each $\boldsymbol{u}_{i}$ belongs to $W^{1, p}\left(\Omega_{i}\right)^{d}$ for some $p>2 d$, and if its norm in this space is small enough with respect to the relative variation of the $\alpha_{i}$, then this solution $\left(\boldsymbol{u}_{i}, p_{i}, k_{i}\right)_{1 \leq i \leq 2}$ is unique. So our idea is to give up making any uniqueness assumption for the analysis of the discretization.

Finally, let us recall the regularity property of the solution which is proved in [3, Thm. 7.5] when the domains $\Omega_{1}$ and $\Omega_{2}$ are two-dimensional rectangles: let $\left(\tilde{U}_{1}, \tilde{U}_{2}\right)$ be any solution of system $(2.4)-(2.5)$, with $\tilde{U}_{i}=\left(\boldsymbol{u}_{i}, p_{i}, k_{i}\right)$, such that $\boldsymbol{u}_{i}, i=1$ and 2 , belong to $H^{s_{-}}\left(\Omega_{i}\right)^{2}$ for some $s_{-}>1$; then, this solution satisfies

$$
\tilde{U}_{i} \in H^{s}\left(\Omega_{i}\right)^{d} \times H^{s-1}\left(\Omega_{i}\right) \times H^{s}\left(\Omega_{i}\right), \quad i=1 \text { and } 2,
$$

for all $s \leq s_{0} \simeq 1.5946$, where the value of $s_{0}$ is derived from [20, Cor. 4.2]. So the following assumption seems reasonable, especially in dimension $d=2$.

Hypothesis 2.2. System (2.4) and (2.5) admits a solution $\left(\tilde{U}_{1}^{*}, \tilde{U}_{2}^{*}\right)$ such that each $\tilde{U}_{i}^{*}, 1 \leq i \leq 2$, belongs to $H^{s^{*}}\left(\Omega_{i}\right)^{d} \times H^{s^{*}-1}\left(\Omega_{i}\right) \times H^{s^{*}}\left(\Omega_{i}\right)$ for some $s^{*}>\frac{d}{2}$.

Remark 2.3. Assume that the functions $\boldsymbol{u}_{i}, i=1$ and 2 , belong to $H^{s}\left(\Omega_{i}\right)^{d}$, for some $s>\frac{d}{2}$. If a solution $k_{i}$ of problem (2.5) belongs to $H^{1}\left(\Omega_{i}\right)$, then it satisfies the more standard formulation of this problem:

Find $k_{i}$ in $H^{1}\left(\Omega_{i}\right), 1 \leq i \leq 2$, with

$$
k_{i}=0 \quad \text { on } \Gamma_{i} \quad \text { and } \quad k_{i}=\left|\boldsymbol{u}_{1}-\boldsymbol{u}_{2}\right|^{2} \quad \text { on } \Gamma,
$$

such that, for $1 \leq i \leq 2$,

$$
\forall \varphi_{i} \in H_{0}^{1}\left(\Omega_{i}\right), \quad c_{i}\left(k_{i} ; k_{i}, \varphi_{i}\right)=\int_{\Omega_{i}} \alpha_{i}\left(k_{i}\right)\left|\nabla \boldsymbol{u}_{i}\right|^{2} \varphi_{i} d \boldsymbol{x},
$$

where the bilinear form $c_{i}\left(t_{i} ; \cdot, \cdot\right)$ is defined by

$$
c_{i}\left(t_{i} ; \ell_{i}, \varphi_{i}\right)=\int_{\Omega_{i}} \gamma_{i}\left(t_{i}\right) \nabla \ell_{i} \cdot \nabla \varphi_{i} d \boldsymbol{x} .
$$


The discretization below relies on this last formulation. However, for technical reasons, we consider in what follows its extension to the case where $k_{i}$ is sought for in $H^{1-\varepsilon}\left(\Omega_{i}\right)$ and $\varphi_{i}$ runs through $H^{1+\varepsilon}\left(\Omega_{i}\right)$ for a small positive $\varepsilon$.

Another presentation. For $1 \leq i \leq 2$, we first introduce a generalized Laplace operator, which we still denote by $\mathcal{L}_{i}$ for simplicity: for a fixed $t_{i}$ in $L^{1}\left(\Omega_{i}\right)$, the operator $\mathcal{L}_{i}\left(t_{i}\right)$ associates with any $g_{i}$ in $L^{1}\left(\Omega_{i}\right)$ and $\lambda_{i}$ in $L^{2}(\Gamma)$ the solution $k_{i}=$ $\mathcal{L}_{i}\left(t_{i}\right)\left(g_{i}, \lambda_{i}\right)$ in $H^{s}\left(\Omega_{i}\right), s<\frac{1}{2}$, defined by transposition, of the problem

$$
\begin{cases}-\operatorname{div}\left(\gamma_{i}\left(t_{i}\right) \nabla k_{i}\right)=g_{i} & \text { in } \Omega_{i}, \\ k_{i}=0 & \text { on } \Gamma_{i}, \\ k_{i}=\lambda_{i} & \text { on } \Gamma, 1 \leq i \leq 2 .\end{cases}
$$

The existence and uniqueness of this solution are checked for instance in [3, sect. 4]. Similarly, we introduce the Stokes operator $\mathcal{S}_{i}$ : for a fixed $t_{i}$ in $L^{1}\left(\Omega_{i}\right)$, the operator $\mathcal{S}_{i}\left(t_{i}\right)$ associates with any $\boldsymbol{g}_{i}$ in the dual space of $X_{i}$ and $\boldsymbol{\lambda}_{i}$ in the dual space of $H_{00}^{\frac{1}{2}}(\Gamma)$ the solution $\boldsymbol{u}_{i}=\mathcal{S}_{i}\left(t_{i}\right)\left(\boldsymbol{g}_{i}, \boldsymbol{\lambda}_{i}\right)$ in $V_{i}$ of the Stokes problem

$$
\begin{cases}-\operatorname{div}\left(\alpha_{i}\left(t_{i}\right) \nabla \boldsymbol{u}_{i}\right)+\operatorname{grad} p_{i}=\boldsymbol{g}_{i} & \text { in } \Omega_{i}, \\ \operatorname{div} \boldsymbol{u}_{i}=0 & \text { in } \Omega_{i}, \\ \boldsymbol{u}_{i}=\mathbf{0} & \text { on } \Gamma_{i}, \\ \alpha_{i}\left(t_{i}\right) \partial_{n_{i}} \boldsymbol{u}_{i}-p_{i} \boldsymbol{n}_{i}=\boldsymbol{\lambda}_{i} & \text { on } \Gamma .\end{cases}
$$

Next it is readily checked that problem (1.1) can be written as

$$
\left(\begin{array}{c}
\boldsymbol{u}_{1} \\
k_{1} \\
\boldsymbol{u}_{2} \\
k_{2}
\end{array}\right)+\left(\begin{array}{cccc}
\mathcal{S}_{1}\left(k_{1}\right) & 0 & 0 & 0 \\
0 & \mathcal{L}_{1}\left(k_{1}\right) & 0 & 0 \\
0 & 0 & \mathcal{S}_{2}\left(k_{2}\right) & 0 \\
0 & 0 & 0 & \mathcal{L}_{2}\left(k_{2}\right)
\end{array}\right)\left(\begin{array}{c}
\left(-\boldsymbol{f}_{1}, \boldsymbol{\lambda}_{1}\left(\boldsymbol{u}_{1}, \boldsymbol{u}_{2}\right)\right) \\
\left(-g_{1}\left(k_{1}, \boldsymbol{u}_{1}\right), \lambda\left(\boldsymbol{u}_{1}, \boldsymbol{u}_{2}\right)\right) \\
\left(-\boldsymbol{f}_{2}, \boldsymbol{\lambda}_{2}\left(\boldsymbol{u}_{1}, \boldsymbol{u}_{2}\right)\right) \\
\left(-g_{2}\left(k_{2}, \boldsymbol{u}_{2}\right), \lambda\left(\boldsymbol{u}_{1}, \boldsymbol{u}_{2}\right)\right)
\end{array}\right)=0
$$

with

$$
\begin{gathered}
\boldsymbol{\lambda}_{i}\left(\boldsymbol{u}_{1}, \boldsymbol{u}_{2}\right)=\left(\boldsymbol{u}_{i}-\boldsymbol{u}_{j}\right)\left|\boldsymbol{u}_{i}-\boldsymbol{u}_{j}\right|, \quad g_{i}\left(k_{i}, \boldsymbol{u}_{i}\right)=\alpha_{i}\left(k_{i}\right)\left|\nabla \boldsymbol{u}_{i}\right|^{2}, \\
\lambda\left(\boldsymbol{u}_{1}, \boldsymbol{u}_{2}\right)=-\left|\boldsymbol{u}_{1}-\boldsymbol{u}_{2}\right|^{2} .
\end{gathered}
$$

Let $\mathcal{T}\left(k_{1}, k_{2}\right)$ denote the diagonal matrix made of the operators $\mathcal{S}_{i}\left(k_{i}\right)$ and $\mathcal{L}_{i}\left(k_{i}\right)$ that appears in (2.15), and let $\mathcal{G}\left(U_{1}, U_{2}\right)$ stand for the last vector in this formula. For technical reasons, we introduce a small parameter $\varepsilon, 0<\varepsilon<\frac{1}{2}$, and we consider the spaces

$$
\mathcal{X}_{i}=X_{i} \times H^{1-\varepsilon}\left(\Omega_{i}\right), \quad \mathcal{X}=\mathcal{X}_{1} \times \mathcal{X}_{2} .
$$

Then problem (1.1) is equivalent to finding a solution $\left(U_{1}, U_{2}\right)$ in $\mathcal{X}$ of the equation

$$
\left(\begin{array}{c}
U_{1} \\
U_{2}
\end{array}\right)+\mathcal{T}\left(k_{1}, k_{2}\right) \mathcal{G}\left(U_{1}, U_{2}\right)=0
$$


In view of [10], we work with a solution $\left(U_{1}^{*}, U_{2}^{*}\right)$ of $(2.18)$ which satisfies the following hypothesis, as usual for the discretization of nonlinear problems.

Hypothesis 2.4. The solution $\left(U_{1}^{*}, U_{2}^{*}\right)$, of system $(2.5)-(2.7)$, with each $U_{i}^{*}=$ $\left(\boldsymbol{u}_{i}^{*}, k_{i}^{*}\right)$, is such that the operator

$$
\mathrm{Id}+D \mathcal{T}\left(k_{1}^{*}, k_{2}^{*}\right) \mathcal{G}\left(U_{1}^{*}, U_{2}^{*}\right)+\mathcal{T}\left(k_{1}^{*}, k_{2}^{*}\right) D \mathcal{G}\left(U_{1}^{*}, U_{2}^{*}\right)
$$

(where $D$ stands for the differential operator) is an isomorphism of $\mathcal{X}$.

The idea is that the conditions for the global uniqueness of the solution $\left(U_{1}^{*}, U_{2}^{*}\right)$, if they exist, are most often too restrictive (see [3, Thm. 6.3]). Hypothesis 2.4 ensures only the local uniqueness of the solution, which is much weaker. Indeed the analogous assumption for the standard Navier-Stokes equations is often used for the numerical analysis of the discretization and is not at all restrictive. Note that Hypothesis 2.4 is equivalent to the well-posedness of the linearized system for any data $\left(\boldsymbol{g}_{i}, \boldsymbol{\lambda}_{i}\right)$ in the dual space of $X_{i} \times H_{00}^{\frac{1}{2}}(\Gamma)$ and $\left(g_{i}, \lambda_{i}\right)$ in $H^{-1-\varepsilon}\left(\Omega_{i}\right) \times H^{\frac{1}{2}-\varepsilon}(\Gamma)$ :

Find $\left(\boldsymbol{w}_{i}, r_{i}\right)$ in $X_{i} \times L^{2}\left(\Omega_{i}\right), 1 \leq i \leq 2$, such that, for $1 \leq i \neq j \leq 2$,

$\forall \boldsymbol{v}_{i} \in X_{i}$

$$
\begin{gathered}
\int_{\Omega_{i}} \alpha_{i}\left(k_{i}^{*}\right) \nabla \boldsymbol{w}_{i}: \nabla \boldsymbol{v}_{i} d \boldsymbol{x}+\int_{\Omega_{i}} \alpha_{i}^{\prime}\left(k_{i}^{*}\right) \ell_{i} \nabla \boldsymbol{u}_{i}^{*}: \nabla \boldsymbol{v}_{i} d \boldsymbol{x}-\int_{\Omega_{i}} r_{i}\left(\operatorname{div} \boldsymbol{v}_{i}\right) d \boldsymbol{x} \\
+\int_{\Gamma} \frac{\left(\boldsymbol{u}_{i}^{*}-\boldsymbol{u}_{j}^{*}\right) \cdot\left(\boldsymbol{w}_{i}-\boldsymbol{w}_{j}\right)}{\left|\boldsymbol{u}_{i}^{*}-\boldsymbol{u}_{j}^{*}\right|}\left(\boldsymbol{u}_{i}^{*}-\boldsymbol{u}_{j}^{*}\right) \cdot \boldsymbol{v}_{i} d \tau+\int_{\Gamma}\left|\boldsymbol{u}_{i}^{*}-\boldsymbol{u}_{j}^{*}\right|\left(\boldsymbol{w}_{i}-\boldsymbol{w}_{j}\right) \cdot \boldsymbol{v}_{i} d \tau \\
=\int_{\Omega_{i}} \boldsymbol{g}_{i} \cdot \boldsymbol{v}_{i} d \boldsymbol{x}+\int_{\Gamma} \boldsymbol{\lambda}_{i} \cdot \boldsymbol{v}_{i} d \tau
\end{gathered}
$$

$\forall q_{i} \in L^{2}\left(\Omega_{i}\right), \quad-\int_{\Omega_{i}} q_{i}\left(\operatorname{div} \boldsymbol{w}_{i}\right) d \boldsymbol{x}=0 ;$

Find $\ell_{i}$ in $H^{1-\varepsilon}\left(\Omega_{i}\right), 1 \leq i \leq 2$, with

$\ell_{i}=0 \quad$ on $\Gamma_{i} \quad$ and $\quad \ell_{i}=\lambda_{i}+2\left(\boldsymbol{u}_{1}^{*}-\boldsymbol{u}_{2}^{*}\right) \cdot\left(\boldsymbol{w}_{1}-\boldsymbol{w}_{2}\right)$ on $\Gamma$,

such that, for $1 \leq i \leq 2$,

$$
\begin{aligned}
& \forall \varphi_{i} \in H_{0}^{1+\varepsilon}\left(\Omega_{i}\right), \\
& \int_{\Omega_{i}} \gamma_{i}\left(k_{i}^{*}\right) \nabla \ell_{i} \cdot \nabla \varphi_{i} d \boldsymbol{x}+\int_{\Omega_{i}} \gamma_{i}^{\prime}\left(k_{i}^{*}\right) \ell_{i} \nabla k_{i}^{*} \cdot \nabla \varphi_{i} d \boldsymbol{x}=\int_{\Omega_{i}} g_{i} \varphi_{i} d \boldsymbol{x} \\
& +2 \int_{\Omega_{i}} \alpha_{i}\left(k_{i}^{*}\right) \nabla \boldsymbol{u}_{i}^{*}: \nabla \boldsymbol{w}_{i} \varphi_{i} d \boldsymbol{x}+\int_{\Omega_{i}} \alpha_{i}^{\prime}\left(k_{i}^{*}\right) \ell_{i}\left|\nabla \boldsymbol{u}_{i}^{*}\right|^{2} \varphi_{i} d \boldsymbol{x} .
\end{aligned}
$$

Even if this is nothing but a linear problem, writing it is rather technical.

In what follows, we always assume that Hypotheses 2.2 and 2.4 hold. These assumptions are nearly realistic and seem necessary for proving the convergence of any type of discretization.

3. Description of the discrete problem. From now on, we assume that the $\Omega_{i}$ are rectangles in the case $d=2$, and rectangular parallelepipeds in the case $d=3$. More precisely, as illustrated in Figure 1, by an appropriate scaling, we take $\Omega_{1}$ (resp., $\Omega_{2}$ ) equal to $]-1,1\left[^{d-1} \times\right] 0, h_{1}[$ (resp., $]-1,1\left[{ }^{d-1} \times\right]-h_{2}, 0\left[\right.$ ), where the $h_{i}$ are positive real numbers. As already said, the $h_{i}$ are often small in practical situations. 


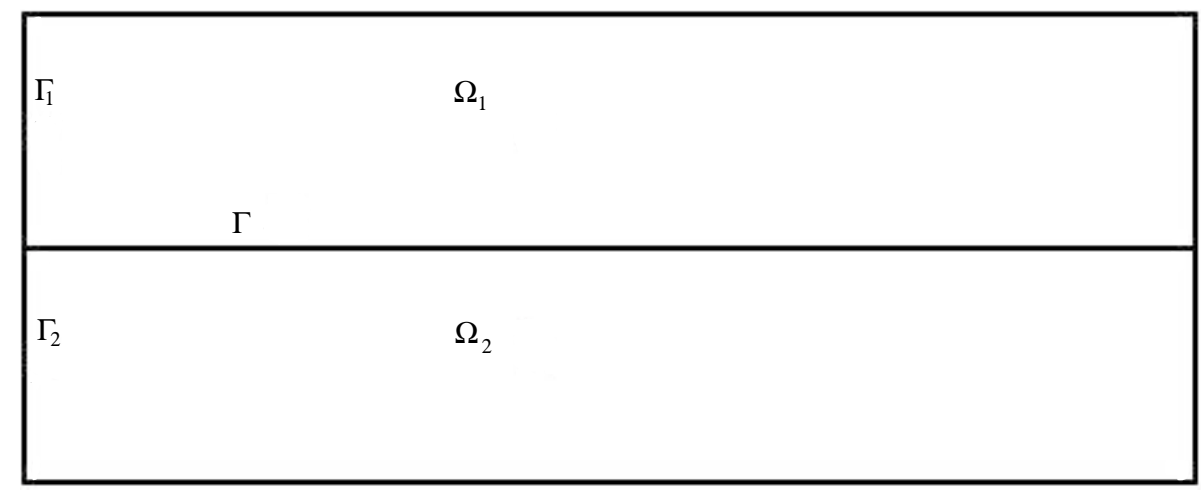

FIG. 1.

We first describe the discrete problem. Second, as for the continuous problem, we write it in a different form, in order to apply the theory of Brezzi, Rappaz, and Raviart [10] for its numerical analysis.

The discrete problem. For each pair of nonnegative integers $(k, n)$, we introduce the space $\mathbb{P}_{k, n}\left(\Omega_{i}\right)$ of restrictions to $\Omega_{i}$ of polynomials with degree $\leq k$ with respect to $x$ (and also to $y$ in the case $d=3$ ) and with degree $\leq n$ with respect to $z$. We denote by $\mathbb{P}_{k}(\Gamma)$ the space of restrictions to $\Gamma$ of polynomials with degree $\leq k$ with respect to each tangential variable. We fix a 4 -tuple $\delta=\left(K_{1}, N_{1}, K_{2}, N_{2}\right)$ of positive integers, in order to define the discrete spaces of velocities and turbulent energies,

$$
X_{i \delta}=\mathbb{P}_{K_{i}, N_{i}}\left(\Omega_{i}\right)^{d} \cap X_{i}, \quad Y_{i \delta}=\mathbb{P}_{K_{i}, N_{i}}\left(\Omega_{i}\right) \cap H_{0}^{1}\left(\Omega_{i}\right) .
$$

As for the standard Stokes problem, two different choices exist for the discrete spaces of pressures $M_{i \delta}$, namely

$$
M_{i \delta}^{1}=\mathbb{P}_{K_{i}-2, N_{i}-2}\left(\Omega_{i}\right) \quad \text { and } \quad M_{i \delta}^{2}=\mathbb{P}_{K_{i}-2, N_{i}-2}\left(\Omega_{i}\right) \cap \mathbb{P}_{\left[\lambda K_{i}\right],\left[\lambda N_{i}\right]}\left(\Omega_{i}\right),
$$

for a parameter $\lambda, 0<\lambda<1$, where the brackets [.] denote the integral part.

We denote by $\left(L_{n}\right)_{n \geq 0}$ the orthogonal basis of $L^{2}(-1,1)$ made by the Legendre polynomials. Each $L_{n}$ has degree $n$ and satisfies $L_{n}(1)=1$. For any positive integer $n$, let $\xi_{j}^{n}$ and $\rho_{j}^{n}, 0 \leq j \leq n$, be the nodes (in increasing order) and weights of the GaussLobatto formula on ] $-1,1$, which is exact on all polynomials with degree $\leq 2 n-1$. We recall that $\xi_{0}^{n}$ (resp., $\xi_{n}^{n}$ ) is equal to -1 (resp., 1 ), that the $\xi_{j}^{n}, 1 \leq j \leq n-1$, are the zeros of $L_{n}^{\prime}$, and that the $\rho_{j}^{n}$ are given by

$$
\rho_{j}^{n}=\frac{2}{n(n+1) L_{n}^{2}\left(\xi_{j}^{n}\right)}, \quad 0 \leq j \leq n .
$$

For simplicity, we denote by $x_{i k}$ and $\rho_{i k}, 0 \leq k \leq K_{i}$, the nodes $\xi_{k}^{K_{i}}$ and weights $\rho_{k}^{K_{i}}$. In the $z$-direction, we set, for $0 \leq j \leq N_{i}$,

$$
z_{i j}=\frac{h_{i}}{2}\left((-1)^{i+1}+\xi_{j}^{N_{i}}\right) \quad \text { and } \quad \omega_{i j}=\frac{h_{i}}{2} \rho_{j}^{N_{i}} .
$$

We introduce the grids

$$
\Xi_{i \delta}= \begin{cases}\left\{\left(x_{i k}, z_{i j}\right) ; 0 \leq k \leq K_{i}, 0 \leq j \leq N_{i}\right\} & \text { in the case } d=2, \\ \left\{\left(x_{i k}, x_{i \ell}, z_{i j}\right) ; 0 \leq k, \ell \leq K_{i}, 0 \leq j \leq N_{i}\right\} & \text { in the case } d=3\end{cases}
$$


and we denote by $\mathcal{I}_{i \delta}$ the Lagrange interpolation operator on the grid $\Xi_{i \delta}$ with values in $\mathbb{P}_{K_{i}, N_{i}}\left(\Omega_{i}\right)$. Two different grids are then defined on the interface $\Gamma$ : we denote by $\mathcal{I}_{i \delta}^{\Gamma}$ the Lagrange interpolation operator on the grid $\Xi_{i \delta} \cap \Gamma$ with values in $\mathbb{P}_{K_{i}}(\Gamma)$. on $\bar{\Omega}_{i}$,

Finally, we introduce the discrete product, for all functions $u$ and $v$ continuous

$$
(u, v)_{i \delta}= \begin{cases}\sum_{k=0}^{K_{i}} \sum_{j=0}^{N_{i}} u\left(x_{i k}, z_{i j}\right) v\left(x_{i k}, z_{i j}\right) \rho_{i k} \omega_{i j} & \text { in the case } d=2, \\ \sum_{k=0}^{K_{i}} \sum_{\ell=0}^{K_{i}} \sum_{j=0}^{N_{i}} u\left(x_{i k}, x_{i \ell}, z_{i j}\right) v\left(x_{i k}, x_{i \ell}, z_{i j}\right) \rho_{i k} \rho_{i \ell} \omega_{i j} & \text { in the case } d=3,\end{cases}
$$

and its analogue on $\Gamma$

$$
(u, v)_{i \delta}^{\Gamma}= \begin{cases}\sum_{k=0}^{K_{i}} u\left(x_{i k}\right) v\left(x_{i k}\right) \rho_{i k} & \text { in the case } d=2, \\ \sum_{k=0}^{K_{i}} \sum_{\ell=0}^{K_{i}} u\left(x_{i k}, x_{i \ell}\right) v\left(x_{i k}, x_{i \ell}\right) \rho_{i k} \rho_{i \ell} & \text { in the case } d=3 .\end{cases}
$$

We fix an operator $\Pi_{i \delta}^{\Gamma}$ from $H_{00}^{\frac{1}{2}}(\Gamma)$ into $\mathbb{P}_{K_{i}}(\Gamma) \cap H_{00}^{\frac{1}{2}}(\Gamma)$ which will be made precise later on. We are now in a position to state the discrete problem associated with problem (1.1). It reads as follows:

Find $\left(\tilde{U}_{1 \delta}, \tilde{U}_{2 \delta}\right)$, with each $\tilde{U}_{i \delta}=\left(\boldsymbol{u}_{i \delta}, p_{i \delta}, k_{i \delta}\right)$ in $X_{i \delta} \times M_{i \delta} \times \mathbb{P}_{K_{i}, N_{i}}\left(\Omega_{i}\right)$, such that, for $1 \leq i \neq j \leq 2$,

$$
k_{i \delta}=0 \quad \text { on } \Gamma_{i} \quad \text { and } \quad k_{i \delta}=\Pi_{i \delta}^{\Gamma}\left(\left|\boldsymbol{u}_{1 \delta}-\boldsymbol{u}_{2 \delta}\right|^{2}\right) \quad \text { on } \Gamma,
$$

and

$$
\begin{aligned}
& \forall \boldsymbol{v}_{i \delta} \in X_{i \delta}, \\
& \quad a_{i \delta}\left(k_{i \delta} ; \boldsymbol{u}_{i \delta}, \boldsymbol{v}_{i \delta}\right)+b_{i \delta}\left(\boldsymbol{v}_{i \delta}, p_{i \delta}\right)+\left(\left|\boldsymbol{u}_{i \delta}-\boldsymbol{u}_{j \delta}\right|\left(\boldsymbol{u}_{i \delta}-\boldsymbol{u}_{j \delta}\right), \boldsymbol{v}_{i \delta}\right)_{i \delta}^{\Gamma}=\left(\boldsymbol{f}_{i}, \boldsymbol{v}_{i \delta}\right)_{i \delta}, \\
& \forall q_{i \delta} \in M_{i \delta}, \quad b_{i \delta}\left(\boldsymbol{u}_{i \delta}, q_{i \delta}\right)=0, \\
& \forall \varphi_{i \delta} \in Y_{i \delta}, \quad c_{i \delta}\left(k_{i \delta} ; k_{i \delta}, \varphi_{i \delta}\right)=\left(\alpha_{i}\left(k_{i \delta}\right)\left|\nabla \boldsymbol{u}_{i \delta}\right|^{2}, \varphi_{i \delta}\right)_{i \delta},
\end{aligned}
$$

where, for any continuous function $t_{i}$, the bilinear forms $a_{i \delta}\left(t_{i} ; \cdot, \cdot\right), b_{i \delta}(\cdot, \cdot)$, and $c_{i \delta}\left(t_{i} ; \cdot, \cdot\right)$ are now defined by

$$
\begin{gathered}
a_{i \delta}\left(t_{i} ; \boldsymbol{u}_{i \delta}, \boldsymbol{v}_{i \delta}\right)=\left(\alpha_{i}\left(t_{i}\right) \nabla \boldsymbol{u}_{i \delta}, \nabla \boldsymbol{v}_{i \delta}\right)_{i \delta}, \quad b_{i \delta}\left(\boldsymbol{v}_{i \delta}, q_{i \delta}\right)=-\left(q_{i \delta}, \operatorname{div} \boldsymbol{v}_{i \delta}\right)_{i \delta}, \\
c_{i \delta}\left(t_{i} ; k_{i \delta}, \varphi_{i \delta}\right)=\left(\gamma_{i}\left(t_{i}\right) \nabla k_{i \delta}, \nabla \varphi_{i \delta}\right)_{i \delta} .
\end{gathered}
$$

Remark 3.1. A natural choice of operator $\Pi_{i \delta}^{\Gamma}$ would be the Lagrange interpolation operator $\mathcal{I}_{i \delta}^{\Gamma}$. However, for $K_{1} \neq K_{2}$, since two different discrete products are defined on the interface $\Gamma$, the trace of $\boldsymbol{u}_{1 \delta}$ on $\Gamma$ must be re-interpolated on the nodes of $\Xi_{2 \delta} \cap \Gamma$ and conversely. Moreover, the convergence of the interpolate of a function $\varphi$ toward this function in $H_{00}^{\frac{1}{2}}(\Gamma)$ or even in $H^{\frac{1}{2}-\varepsilon}(\Gamma)$ would require too much regularity of the function $\varphi$; see [7, sect. 14]. Other choices of operator $\Pi_{i \delta}^{\Gamma}$, such as orthogonal projection operators, are possible but seem more expensive to implement.

Remark 3.2. For the choices $M_{i \delta}^{1}$ and $M_{i \delta}^{2}$ of discrete pressure spaces introduced in (3.2), and thanks to the exactness property of the quadrature formula, each $b_{i \delta}(\cdot, \cdot)$ can be replaced by $b_{i}(\cdot, \cdot)$ in formulation $(3.5)$.

The numerical analysis of system (3.4)-(3.5) is rather technical. However, we begin with the same simplication as for the continuous problem. For $i=1$ and 2 , we introduce the discrete kernel

$$
V_{i \delta}=\left\{\boldsymbol{v}_{i \delta} \in X_{i \delta} ; \forall q_{i \delta} \in M_{i \delta}, b_{i \delta}\left(\boldsymbol{v}_{i \delta}, q_{i \delta}\right)=0\right\} .
$$


Note that, for the two choices $M_{i \delta}=M_{i \delta}^{1}$ and $M_{i \delta}=M_{i \delta}^{2}$ proposed in (3.2), $V_{i \delta}$ is not contained in $V_{i}$, i.e., is not made of exactly divergence-free polynomials. It is readily checked with this definition that, for each pair $\left(\tilde{U}_{1 \delta}, \tilde{U}_{2 \delta}\right)$ solution of system (3.4)-(3.5), the reduced pair $\left(U_{1 \delta}, U_{2 \delta}\right)$ of discrete velocities and discrete turbulent energies is a solution of the following system:

Find $\left(U_{1 \delta}, U_{2 \delta}\right)$, with each $U_{i \delta}=\left(\boldsymbol{u}_{i \delta}, k_{i \delta}\right)$ in $V_{i \delta} \times \mathbb{P}_{K_{i}, N_{i}}\left(\Omega_{i}\right)$, satisfying (3.4) and such that, for $1 \leq i \neq j \leq 2$,

$$
\begin{aligned}
& \forall \boldsymbol{v}_{i \delta} \in V_{i \delta}, \quad a_{i \delta}\left(k_{i \delta} ; \boldsymbol{u}_{i \delta}, \boldsymbol{v}_{i \delta}\right)+\left(\left|\boldsymbol{u}_{i \delta}-\boldsymbol{u}_{j \delta}\right|\left(\boldsymbol{u}_{i \delta}-\boldsymbol{u}_{j \delta}\right), \boldsymbol{v}_{i \delta}\right)_{i \delta}^{\Gamma}=\left(\boldsymbol{f}_{i}, \boldsymbol{v}_{i \delta}\right)_{i \delta}, \\
& \forall \varphi_{i \delta} \in Y_{i \delta}, \quad c_{i \delta}\left(k_{i \delta} ; k_{i \delta}, \varphi_{i \delta}\right)=\left(\alpha_{i}\left(k_{i \delta}\right)\left|\nabla \boldsymbol{u}_{i \delta}\right|^{2}, \varphi_{i \delta}\right)_{i \delta} .
\end{aligned}
$$

The converse property relies on a discrete inf-sup condition, which is derived in two steps, relying on the arguments in [8] and [9], respectively. For a while, let $\tilde{M}_{i \delta}^{m}$ stand for the subspace of $M_{i \delta}^{m}$ made of polynomials with a null integral on $\Omega_{i}$.

Lemma 3.3. For $i=1$ and 2 , and for the discrete spaces $\tilde{M}_{i \delta}^{m}, m=1$ and 2 , there exists a constant $\tilde{\beta}_{i \delta}^{m}>0$ such that

$$
\forall q_{i \delta} \in \tilde{M}_{i \delta}^{m}, \quad \sup _{\boldsymbol{v}_{i \delta} \in X_{i \delta} \cap H_{0}^{1}\left(\Omega_{i}\right)^{d}} \frac{b_{i \delta}\left(\boldsymbol{v}_{i \delta}, q_{i \delta}\right)}{\left\|\boldsymbol{v}_{i \delta}\right\|_{H^{1}\left(\Omega_{i}\right)^{d}}} \geq \tilde{\beta}_{i \delta}^{m}\left\|q_{i \delta}\right\|_{L^{2}\left(\Omega_{i}\right)} .
$$

Moreover, these constants $\tilde{\beta}_{i}^{m}$ satisfy, for $i=1$ and 2 ,

$$
\tilde{\beta}_{i}^{1} \geq c K_{i}^{\frac{2-d}{2}} \inf \left\{K_{i}^{-\frac{1}{2}}, N_{i}^{-\frac{1}{2}}\right\} \quad \text { and } \quad \tilde{\beta}_{i}^{2} \geq c .
$$

Proof. Since any $q_{i \delta}$ in $\tilde{M}_{i \delta}$ has a null integral on $\Omega_{i}$, there exists [15, Chap. I, Cor. 2.4] a function $\boldsymbol{v}_{i}$ in $H_{0}^{1}\left(\Omega_{i}\right)^{d}$ such that

$$
\operatorname{div} \boldsymbol{v}_{i}=q_{i \delta} \quad \text { in } \Omega_{i} \quad \text { and } \quad\left\|\boldsymbol{v}_{i}\right\|_{H^{1}\left(\Omega_{i}\right)^{d}} \leq c\left\|q_{i \delta}\right\|_{L^{2}\left(\Omega_{i}\right)} .
$$

Next, we recall from [8, Lems. 3.2 and 3.3] that, for any $\mu, 0<\mu<1$, there exists an operator $\pi_{n}^{\mu}$ from $H_{0}^{1}(-1,1)$ onto $\mathbb{P}_{[(1+\mu) n]}(-1,1) \cap H_{0}^{1}(-1,1)$ which preserves all polynomials in $\mathbb{P}_{n-1}(-1,1)$ and satisfies, for all $\varphi$ in $H_{0}^{1}(-1,1)$,

$$
\left\|\left(\pi_{n}^{\mu} \varphi\right)^{\prime}\right\|_{L^{2}(-1,1)} \leq\left\|\varphi^{\prime}\right\|_{L^{2}(-1,1)} \quad \text { and } \quad\left\|\pi_{n}^{\mu} \varphi\right\|_{L^{2}(-1,1)} \leq c \mu^{-\frac{1}{2}}\|\varphi\|_{L^{2}(-1,1)} .
$$

The idea consists of choosing the operator $\pi_{K_{i}}^{m}$ in the $x$ - or $y$-direction equal to $\pi_{M}^{\mu\left(K_{i}\right)}$, with

$$
\left(1+\mu\left(K_{i}\right)\right) M=K_{i} \quad \text { and } \quad M= \begin{cases}K_{i}-1 & \text { if } m=1, \\ {\left[\frac{1+\lambda}{2} K_{i}\right]} & \text { if } m=2\end{cases}
$$

(recall that $\lambda$ is introduced in (3.2)), and denoting them by $\pi_{K_{i}}^{m(x)}$ and $\pi_{K_{i}}^{m(y)}$, respectively. Similarly, the operator $\pi_{N_{i}}^{m}$ in the $z$-direction is equal to $\pi_{M}^{\mu\left(N_{i}\right)}$, with

$$
\left(1+\mu\left(N_{i}\right)\right) M=N_{i} \quad \text { and } \quad M= \begin{cases}N_{i}-1 & \text { if } m=1, \\ {\left[\frac{1+\lambda}{2} N_{i}\right]} & \text { if } m=2,\end{cases}
$$

and denoted by $\pi_{N_{i}}^{m(z)}$. Next we set

$$
\boldsymbol{v}_{i \delta}^{s}= \begin{cases}\pi_{K_{i}}^{m(x)} \circ \pi_{N_{i}}^{m(z)} \boldsymbol{v}_{i} & \text { in the case } d=2, \\ \pi_{K_{i}}^{m(x)} \circ \pi_{K_{i}}^{m(y)} \circ \pi_{N_{i}}^{m(z)} \boldsymbol{v}_{i} & \text { in the case } d=3 .\end{cases}
$$


From the properties of these operators, it is readily checked that

$$
b_{i \delta}\left(\boldsymbol{v}_{i \delta}, q_{i \delta}\right)=-\int_{\Omega_{i}} q_{i \delta}\left(\operatorname{div} \boldsymbol{v}_{i}\right) d \boldsymbol{x}=\int_{\Omega_{i \delta}}\left(q_{i \delta}\right)^{2} d \boldsymbol{x},
$$

while the norm of $\boldsymbol{v}_{i \delta}$ in $H_{0}^{1}(\Omega)^{d}$ is bounded by

$$
\left\|\boldsymbol{v}_{i \delta}\right\|_{H^{1}\left(\Omega_{i}\right)^{d}} \leq c \sup \left\{\mu\left(K_{i}\right)^{\frac{2-d}{2}} \mu\left(N_{i}\right)^{-\frac{1}{2}}, \mu\left(K_{i}\right)^{\frac{1-d}{2}}\right\}\left\|\boldsymbol{v}_{i}\right\|_{H^{1}\left(\Omega_{i}\right)^{d}} .
$$

Evaluating the quantities $\mu\left(K_{i}\right)$ and $\mu\left(N_{i}\right)$ as a function of $N_{i}$ or $K_{i}$ for $m=1$ or 2 leads to the desired result.

LEMMA 3.4. For $i=1$ and 2 , and for the discrete spaces $M_{i \delta}^{m}, m=1$ and 2, defined in (3.2), there exists a constant $\beta_{i \delta}^{m}>0$ such that

$$
\forall q_{i \delta} \in M_{i \delta}^{m}, \quad \sup _{\boldsymbol{v}_{i \delta} \in X_{i \delta}} \frac{b_{i \delta}\left(\boldsymbol{v}_{i \delta}, q_{i \delta}\right)}{\left\|\boldsymbol{v}_{i \delta}\right\|_{H^{1}\left(\Omega_{i}\right)^{d}}} \geq \beta_{i \delta}^{m}\left\|q_{i \delta}\right\|_{L^{2}\left(\Omega_{i}\right)} .
$$

Moreover, these constants $\beta_{i \delta}^{m}, i=1$ and 2, satisfy (3.9).

Proof. Any function $q_{i \delta}$ in $M_{i \delta}^{m}$ admits the expansion

$$
q_{i \delta}=\tilde{q}_{i \delta}+\bar{q}_{i \delta}, \quad \text { with } \quad \bar{q}_{i \delta}=\frac{1}{2^{d-1} h_{i}} \int_{\Omega_{i}} q_{i \delta}(\boldsymbol{x}) d \boldsymbol{x} .
$$

Since the function $\tilde{q}_{i \delta}$ belongs to $\tilde{M}_{i \delta}^{m}$, it follows from Lemma 3.3 that there exists a $\tilde{\boldsymbol{v}}_{i \delta}$ in $X_{i \delta} \cap H_{0}^{1}\left(\Omega_{i}\right)^{d}$ such that

$$
b_{i \delta}\left(\tilde{\boldsymbol{v}}_{i \delta}, \tilde{q}_{i \delta}\right)=\left\|\tilde{q}_{i \delta}\right\|_{L^{2}\left(\Omega_{i}\right)}^{2} \quad \text { and } \quad\left\|\tilde{\boldsymbol{v}}_{i \delta}\right\|_{H^{1}\left(\Omega_{i}\right)^{d}} \leq \frac{1}{\tilde{\beta}_{i \delta}^{m}}\left\|\tilde{q}_{i \delta}\right\|_{L^{2}\left(\Omega_{i}\right)} .
$$

On the other hand, the function $\overline{\boldsymbol{v}}_{i \delta}$ equal to $\left(0, v_{i \delta}\right)$ in dimension $d=2$, and to $\left(0,0, v_{i \delta}\right)$ in dimension $d=3$, with

$$
v_{i \delta}= \begin{cases}\left(L_{0}(x)-L_{2}(x)\right)\left((-1)^{i+1} h_{i}-z\right) \bar{q}_{i \delta} & \text { in dimension } d=2, \\ \left(L_{0}(x)-L_{2}(x)\right)\left(L_{0}(y)-L_{2}(y)\right)\left((-1)^{i+1} h_{i}-z\right) \bar{q}_{i \delta} & \text { in dimension } d=3,\end{cases}
$$

belongs to $X_{i \delta}$ and satisfies, for a fixed constant $c_{0}$,

$$
b_{i \delta}\left(\overline{\boldsymbol{v}}_{i \delta}, \bar{q}_{i \delta}\right)=\left\|\bar{q}_{i \delta}\right\|_{L^{2}\left(\Omega_{i}\right)}^{2} \quad \text { and } \quad\left\|\overline{\boldsymbol{v}}_{i \delta}\right\|_{H^{1}\left(\Omega_{i}\right)^{d}} \leq c_{0}\left\|\bar{q}_{i \delta}\right\|_{L^{2}\left(\Omega_{i}\right)} .
$$

Next we take $\boldsymbol{v}_{i \delta}$ equal to $\tilde{\boldsymbol{v}}_{i \delta}+\lambda \overline{\boldsymbol{v}}_{i \delta}$ for a fixed constant $\lambda$. Indeed, it follows by integration by parts that $b_{i \delta}\left(\tilde{\boldsymbol{v}}_{i \delta}, \bar{q}_{i \delta}\right)$ vanishes so that

$$
b_{i \delta}\left(\boldsymbol{v}_{i \delta}, q_{i \delta}\right)=b_{i \delta}\left(\tilde{\boldsymbol{v}}_{i \delta}, \tilde{q}_{i \delta}\right)+\lambda b_{i \delta}\left(\overline{\boldsymbol{v}}_{i \delta}, \bar{q}_{i \delta}\right)+\lambda b_{i \delta}\left(\overline{\boldsymbol{v}}_{i \delta}, \tilde{q}_{i \delta}\right) .
$$

The previous properties, together with the continuity of $b_{i \delta}(\cdot, \cdot)$ (which coincides with $b_{i}(\cdot, \cdot)$ everywhere in the previous equation), yield

$$
\begin{aligned}
b_{i \delta}\left(\boldsymbol{v}_{i \delta}, q_{i \delta}\right) \geq\left\|\tilde{q}_{i \delta}\right\|_{L^{2}\left(\Omega_{i}\right)}^{2}+\lambda\left\|\bar{q}_{i \delta}\right\|_{L^{2}\left(\Omega_{i}\right)}^{2}-c \lambda\left\|\overline{\boldsymbol{v}}_{i \delta}\right\|_{H^{1}\left(\Omega_{i}\right)^{d}}\left\|\tilde{q}_{i \delta}\right\|_{L^{2}\left(\Omega_{i}\right)} \\
\geq\left\|\tilde{q}_{i \delta}\right\|_{L^{2}\left(\Omega_{i}\right)}^{2}+\lambda\left\|\bar{q}_{i \delta}\right\|_{L^{2}\left(\Omega_{i}\right)}^{2}-c c_{0} \lambda\left\|\tilde{q}_{i \delta}\right\|_{L^{2}\left(\Omega_{i}\right)}\left\|\bar{q}_{i \delta}\right\|_{L^{2}\left(\Omega_{i}\right)},
\end{aligned}
$$

whence

$$
b_{i \delta}\left(\boldsymbol{v}_{i \delta}, q_{i \delta}\right) \geq \frac{1}{2}\left\|\tilde{q}_{i \delta}\right\|_{L^{2}\left(\Omega_{i}\right)}^{2}+\lambda\left(1-\frac{c^{2} c_{0}^{2} \lambda}{2}\right)\left\|\bar{q}_{i \delta}\right\|_{L^{2}\left(\Omega_{i}\right)}^{2}
$$


We now choose $\lambda$ equal to $\frac{1}{c^{2} c_{0}^{2}}$, which gives (note that $\tilde{q}_{i \delta}$ and $\bar{q}_{i \delta}$ are orthogonal in $\left.L^{2}\left(\Omega_{i}\right)\right)$

$$
b_{i \delta}\left(\boldsymbol{v}_{i \delta}, q_{i \delta}\right) \geq \inf \left\{\frac{1}{2}, \frac{\lambda}{2}\right\}\left\|q_{i \delta}\right\|_{L^{2}\left(\Omega_{i}\right)}^{2} .
$$

We also have

$$
\left\|\boldsymbol{v}_{i \delta}\right\|_{H^{1}\left(\Omega_{i}\right)^{d}} \leq\left\|\tilde{\boldsymbol{v}}_{i \delta}\right\|_{H^{1}\left(\Omega_{i}\right)^{d}}+\lambda\left\|\overline{\boldsymbol{v}}_{i \delta}\right\|_{H^{1}\left(\Omega_{i}\right)^{d}} \leq\left(\left(\frac{1}{\tilde{\beta}_{i \delta}^{m}}\right)^{2}+c_{0}^{2} \lambda^{2}\right)^{\frac{1}{2}}\left\|q_{i \delta}\right\|_{L^{2}\left(\Omega_{i}\right)},
$$

which concludes the proof.

Remark 3.5. From the previous proofs, the constants $\beta_{i \delta}^{m}$ given in (3.9) a priori depend on $h_{i}$. However, by using the vertical homothety that maps $\Omega_{i}$ onto the reference square or cube, it is readily checked that these constants satisfy

$$
\beta_{i}^{1} \geq c K_{i}^{\frac{2-d}{2}} \inf \left\{h_{i} K_{i}^{-\frac{1}{2}}, N_{i}^{-\frac{1}{2}}\right\} \quad \text { and } \quad \beta_{i}^{2} \geq c h_{i},
$$

where $c$ is now independent of $h_{i}$.

So we now work with system (3.4)-(3.7). The first idea consists of writing it in a more appropriate form which is the discrete analogue of (2.18).

Another presentation. For $i=1$ and 2, we introduce the discrete Laplace operator $\mathcal{L}_{i \delta}$. For a fixed continuous function $t_{i}$, the operator $\mathcal{L}_{i \delta}\left(t_{i}\right)$ associates with any $g_{i}$ in $H^{-1}\left(\Omega_{i}\right)$ and any function $\lambda_{i}$ in $H_{00}^{\frac{1}{2}}(\Gamma)$, the solution $k_{i \delta}=\mathcal{L}_{i \delta}\left(t_{i}\right)\left(g_{i}, \lambda_{i}\right)$ of the following problem:

$$
\begin{aligned}
& \text { Find } k_{i \delta} \text { in } \mathbb{P}_{K_{i}, N_{i}}\left(\Omega_{i}\right) \text { such that } \\
& \qquad k_{i \delta}=0 \quad \text { on } \Gamma_{i} \text { and } k_{i \delta}=\Pi_{i \delta}^{\Gamma} \lambda_{i} \quad \text { on } \Gamma,
\end{aligned}
$$

and

$$
\forall \varphi_{i \delta} \in Y_{i \delta}, \quad c_{i \delta}\left(t_{i} ; k_{i \delta}, \varphi_{i \delta}\right)=\int_{\Omega_{i}} g_{i} \varphi_{i \delta} d \boldsymbol{x} .
$$

It follows from (3.3) that the weights $\rho_{i k}$ and $\omega_{i j}$ are positive. When combined with (2.1), this yields that the only solution of (3.12)-(3.13) for $g_{i}=0$ and $\lambda_{i}=0$ is zero. Hence, since problem (3.12)-(3.13) results into a square linear system, the operator $\mathcal{L}_{i \delta}\left(t_{i}\right)$ is well-defined.

Similarly, we introduce the discrete Stokes operator $\mathcal{S}_{i \delta}$. For a fixed continuous function $t_{i}$, the operator $\mathcal{S}_{i \delta}\left(t_{i}\right)$ associates with any $\boldsymbol{g}_{i}$ in the dual space of $X_{i}$ and $\boldsymbol{\lambda}_{i}$ in the dual space of $H_{00}^{\frac{1}{2}}(\Gamma)^{d}$ the solution $\boldsymbol{u}_{i \delta}=\mathcal{S}_{i \delta}\left(t_{i}\right)\left(\boldsymbol{g}_{i}, \boldsymbol{\lambda}_{i}\right)$ in $V_{i \delta}$ of the following Stokes problem:

Find $\boldsymbol{u}_{i \delta}$ in $V_{i \delta}$ such that

$$
\forall \boldsymbol{v}_{i \delta} \in V_{i \delta}, \quad a_{i \delta}\left(t_{i} ; \boldsymbol{u}_{i \delta}, \boldsymbol{v}_{i \delta}\right)=\int_{\Omega_{i}} \boldsymbol{g}_{i} \cdot \boldsymbol{v}_{i \delta} d \boldsymbol{x}+\int_{\Gamma} \boldsymbol{\lambda}_{i} \cdot \boldsymbol{v}_{i \delta} d \tau .
$$

There also it follows from (3.3) and (2.1) that the operator $\mathcal{S}_{i \delta}\left(t_{i}\right)$ is well-defined.

Finally, the matrix $\mathcal{T}_{\delta}\left(t_{1}, t_{2}\right)$ is defined by

$$
\mathcal{T}_{\delta}\left(t_{1}, t_{2}\right)=\left(\begin{array}{cccc}
\mathcal{S}_{1 \delta}\left(t_{1}\right) & 0 & 0 & 0 \\
0 & \mathcal{L}_{1 \delta}\left(t_{1}\right) & 0 & 0 \\
0 & 0 & \mathcal{S}_{2 \delta}\left(t_{2}\right) & 0 \\
0 & 0 & 0 & \mathcal{L}_{2 \delta}\left(t_{2}\right)
\end{array}\right)
$$


We introduce the vector $\mathcal{G}_{\delta}\left(U_{1 \delta}, U_{2 \delta}\right)$,

$$
\mathcal{G}_{\delta}\left(U_{1 \delta}, U_{2 \delta}\right)=\left(\begin{array}{c}
\left(-\boldsymbol{f}_{1 \delta}, \boldsymbol{\lambda}_{1 \delta}\left(\boldsymbol{u}_{1 \delta}, \boldsymbol{u}_{2 \delta}\right)\right) \\
\left(-g_{1 \delta}\left(k_{1 \delta}, \boldsymbol{u}_{1 \delta}\right), \lambda\left(\boldsymbol{u}_{1 \delta}, \boldsymbol{u}_{2 \delta}\right)\right) \\
\left(-\boldsymbol{f}_{2 \delta}, \boldsymbol{\lambda}_{2 \delta}\left(\boldsymbol{u}_{1 \delta}, \boldsymbol{u}_{2 \delta}\right)\right) \\
\left(-g_{2 \delta}\left(k_{2 \delta}, \boldsymbol{u}_{2 \delta}\right), \lambda\left(\boldsymbol{u}_{1 \delta}, \boldsymbol{u}_{2 \delta}\right)\right)
\end{array}\right),
$$

where the functions $\boldsymbol{f}_{i \delta}, g_{i \delta}$, and $\boldsymbol{\lambda}_{i \delta}$ are defined by duality, for smooth enough functions $\boldsymbol{v}_{i}$ and $\varphi_{i}$ (we do not make precise the spaces),

$$
\begin{gathered}
\left\langle\boldsymbol{f}_{i \delta}, \boldsymbol{v}_{i}\right\rangle=\left(\boldsymbol{f}_{i}, \boldsymbol{v}_{i}\right)_{i \delta}, \quad\left\langle\boldsymbol{\lambda}_{i \delta}\left(\boldsymbol{u}_{1 \delta}, \boldsymbol{u}_{2 \delta}\right), \boldsymbol{v}_{i}\right\rangle=\left(\left|\boldsymbol{u}_{i \delta}-\boldsymbol{u}_{j \delta}\right|\left(\boldsymbol{u}_{i \delta}-\boldsymbol{u}_{j \delta}\right), \boldsymbol{v}_{i}\right)_{i \delta}^{\Gamma}, \\
\left\langle g_{i \delta}\left(k_{i \delta}, \boldsymbol{u}_{i \delta}\right), \varphi_{i}\right\rangle=\left(\alpha_{i}\left(k_{i \delta}\right)\left|\nabla \boldsymbol{u}_{i \delta}\right|^{2}, \varphi_{i}\right)_{i \delta} .
\end{gathered}
$$

The quantity $\lambda\left(\boldsymbol{u}_{1}, \boldsymbol{u}_{2}\right)$ is defined in (2.16).

Thus, it is readily checked that problem (3.4)-(3.7) can be equivalently written

$$
U_{\delta}+\mathcal{T}_{\delta}\left(k_{1 \delta}, k_{2 \delta}\right) \mathcal{G}_{\delta}\left(U_{1 \delta}, U_{2 \delta}\right)=0, \quad \text { with } \quad U_{\delta}=\left(\begin{array}{c}
\boldsymbol{u}_{1 \delta} \\
k_{1 \delta} \\
\boldsymbol{u}_{2 \delta} \\
k_{2 \delta}
\end{array}\right) .
$$

This formulation is fully appropriate for performing its numerical analysis thanks to the theory of Brezzi, Rappaz, and Raviart [10].

4. The discrete Laplace and Stokes operators. As a first step for the numerical analysis of the discrete problem (3.4)-(3.5), we investigate the properties of the discrete quasi-linear operators $\mathcal{L}_{i \delta}$ and $\mathcal{S}_{i \delta}$; more precisely, we prove stability and error estimates. In all that follows, $c, c^{\prime}$, and $c^{\prime \prime}$ stand for generic constants that may vary from one line to the other but are always independent of $\delta$.

The discrete Laplace operator. For $i=1$ and 2, and for a fixed continuous function $t_{i}$, let us first consider the operator $\mathcal{L}_{i \delta}\left(t_{i}\right)$ defined from problem (3.12)(3.13). In order to prove its stability, we first recall [7, Form. (13.10)] that, for any polynomial $\varphi_{n}$ of degree $\leq n$ on $]-1,1[$,

$$
\left\|\varphi_{n}\right\|_{L^{2}(-1,1)}^{2} \leq \sum_{j=0}^{n} \varphi_{n}^{2}\left(\xi_{j}^{n}\right) \rho_{j}^{n} \leq 3\left\|\varphi_{n}\right\|_{L^{2}(-1,1)}^{2} .
$$

Combined with the boundedness and positivity of $\gamma_{i}$ (see (2.1)), this obviously yields some basic properties of the form $c_{i \delta}(\cdot, \cdot)$ that we now state.

LEMma 4.1. For any continuous function $t_{i}$, the form $c_{i \delta}\left(t_{i} ; \cdot, \cdot\right)$ satisfies the following properties of continuity:

$$
\begin{aligned}
\forall \psi_{i \delta} \in \mathbb{P}_{K_{i}, N_{i}}\left(\Omega_{i}\right), \forall \varphi_{i \delta} \in & \mathbb{P}_{K_{i}, N_{i}}\left(\Omega_{i}\right), \\
& c_{i \delta}\left(t_{i} ; \psi_{i \delta}, \varphi_{i \delta}\right) \leq c\left\|\psi_{i \delta}\right\|_{H^{1}\left(\Omega_{i}\right)}\left\|\varphi_{i \delta}\right\|_{H^{1}\left(\Omega_{i}\right)},
\end{aligned}
$$

and of ellipticity

$$
\forall \psi_{i \delta} \in Y_{i \delta}, \quad c_{i \delta}\left(t_{i} ; \psi_{i \delta}, \psi_{i \delta}\right) \geq c\left\|\psi_{i \delta}\right\|_{H^{1}\left(\Omega_{i}\right)}^{2}
$$

Let $R_{i}$ be a continuous lifting operator form $H_{00}^{\frac{1}{2}}(\Gamma)$ into $H^{1}\left(\Omega_{i}\right)$, defined as follows. For any $\lambda$ in $H_{00}^{\frac{1}{2}}(\Gamma), R_{i} \lambda$ belongs to $H^{1}\left(\Omega_{i}\right)$, is equal to $\lambda$ on $\Gamma$, vanishes 
on $\Gamma_{i}$, and satisfies (this is proven by using the analogous lifting operator on the unit square or cube), for all $s \geq 1$,

$$
\forall \lambda \in H_{\diamond}^{s-\frac{1}{2}}(\Gamma), \quad\left\|R_{i} \lambda\right\|_{H^{s}\left(\Omega_{i}\right)} \leq c h_{i}^{\frac{1}{2}-s}\|\lambda\|_{H_{\diamond}^{s-\frac{1}{2}}(\Gamma)},
$$

where $H_{\diamond}^{s-\frac{1}{2}}(\Gamma)$ stands for the intersection $H^{s-\frac{1}{2}}(\Gamma) \cap H_{00}^{\frac{1}{2}}(\Gamma)$, provided with the norm of $H_{00}^{\frac{1}{2}}(\Gamma)$ if $s$ is equal to 1 , of $H^{s-\frac{1}{2}}(\Gamma)$ if $s>1$. A similar operator $R_{i \delta}$, satisfying the same properties, is constructed in [19] and [4], which maps polynomials in $\mathbb{P}_{K_{i}}(\Gamma)$ vanishing on $\partial \Gamma$ into $\mathbb{P}_{K_{i}, K_{i}}\left(\Omega_{i}\right)$. Moreover, this operator satisfies, for all $s \geq 1$,

$$
\forall \lambda_{\delta} \in \mathbb{P}_{K_{i}}(\Gamma) \cap H_{00}^{\frac{1}{2}}(\Gamma), \quad\left\|R_{i \delta} \lambda_{\delta}\right\|_{H^{s}\left(\Omega_{i}\right)} \leq c h_{i}^{\frac{1}{2}-s}\left\|\lambda_{\delta}\right\|_{H_{\diamond}^{s-\frac{1}{2}}(\Gamma)} .
$$

However, in order to obtain a lifting operator of the same space of polynomials into $\mathbb{P}_{K_{i}, N_{i}}(\Gamma)$, we apply the interpolation operator $\mathcal{I}_{i \delta}$ to $R_{i \delta} \lambda_{\delta}$ and derive from the stability properties of this operator on polynomials (see [7, Forms. (13.27) and (13.28)]) that

(4.6) $\forall \lambda_{\delta} \in \mathbb{P}_{K_{i}}(\Gamma) \cap H_{00}^{\frac{1}{2}}(\Gamma), \quad\left\|\mathcal{I}_{i \delta} R_{i \delta} \lambda_{\delta}\right\|_{H^{1}\left(\Omega_{i}\right)} \leq c \sup \left\{h_{i}^{-\frac{1}{2}}, \frac{K_{i}}{N_{i}} h_{i}^{\frac{1}{2}}\right\}\left\|\lambda_{\delta}\right\|_{H_{00}^{\frac{1}{2}}(\Gamma)}$.

LEMma 4.2. For any continuous function $t_{i}$, the following stability property holds for any $g_{i}$ in $H^{-1}\left(\Omega_{i}\right)$ and any continuous function $\lambda_{i}$ on $\Gamma$ :

$$
\left\|\mathcal{L}_{i \delta}\left(t_{i}\right)\left(g_{i}, \lambda_{i}\right)\right\|_{H^{1}\left(\Omega_{i}\right)} \leq c\left(\left\|g_{i}\right\|_{H^{-1}\left(\Omega_{i}\right)}+\sup \left\{h_{i}^{-\frac{1}{2}}, \frac{K_{i}}{N_{i}} h_{i}^{\frac{1}{2}}\right\}\left\|\Pi_{i \delta}^{\Gamma} \lambda_{i}\right\|_{H_{00}^{\frac{1}{2}}(\Gamma)}\right) .
$$

Proof. The function $k_{i \delta}^{0}=\mathcal{L}_{i \delta}\left(t_{i}\right)\left(g_{i}, \lambda_{i}\right)-\mathcal{I}_{i \delta} R_{i \delta} \Pi_{i \delta}^{\Gamma} \lambda_{i}$ belongs to $Y_{i \delta}$ so that applying the ellipticity property (4.3) leads to

$$
c\left\|k_{i \delta}^{0}\right\|_{H^{1}\left(\Omega_{i}\right)}^{2} \leq c_{i \delta}\left(t_{i} ; k_{i \delta}^{0}, k_{i \delta}^{0}\right)=\int_{\Omega_{i}} g_{i} k_{i \delta}^{0} d \boldsymbol{x}-c_{i \delta}\left(t_{i} ; \mathcal{I}_{i \delta} R_{i \delta} \Pi_{i \delta}^{\Gamma} \lambda_{i}, k_{i \delta}^{0}\right) .
$$

The continuity property (4.2) gives

$$
\left\|k_{i \delta}^{0}\right\|_{H^{1}\left(\Omega_{i}\right)} \leq c\left(\left\|g_{i}\right\|_{H^{-1}\left(\Omega_{i}\right)}+\left\|\mathcal{I}_{i \delta} R_{i \delta} \Pi_{i \delta}^{\Gamma} \lambda_{i}\right\|_{H^{1}\left(\Omega_{i}\right)}\right),
$$

whence, by a triangle inequality,

$$
\left\|\mathcal{L}_{i \delta}\left(t_{i}\right)\left(g_{i}, \lambda_{i}\right)\right\|_{H^{1}\left(\Omega_{i}\right)} \leq c\left(\left\|g_{i}\right\|_{H^{-1}\left(\Omega_{i}\right)}+\left\|\mathcal{I}_{i \delta} R_{i \delta} \Pi_{i \delta}^{\Gamma} \lambda_{i}\right\|_{H^{1}\left(\Omega_{i}\right)}\right) .
$$

The desired estimate then follows from (4.6).

Remark 4.3. Note from the previous proof that estimate (4.7) can be replaced by

$$
\left\|\mathcal{L}_{i \delta}\left(t_{i}\right)\left(g_{i}, \lambda_{i}\right)\right\|_{H^{1}\left(\Omega_{i}\right)} \leq c\left(\left\|g_{i}\right\|_{Y_{i \delta}^{\prime}}+\sup \left\{h_{i}^{-\frac{1}{2}}, \frac{K_{i}}{N_{i}} h_{i}^{\frac{1}{2}}\right\}\left\|\Pi_{i \delta}^{\Gamma} \lambda_{i}\right\|_{H_{00}^{\frac{1}{2}}(\Gamma)}\right),
$$

where the dual norm $\|\cdot\|_{Y_{i \delta}^{\prime}}$ is defined in a trivial way by

$$
\left\|g_{i}\right\|_{Y_{i \delta}^{\prime}}=\sup _{\varphi_{i \delta} \in Y_{i \delta}} \frac{\int_{\Omega_{i}} g_{i} \varphi_{i \delta} d \boldsymbol{x}}{\left\|\varphi_{i \delta}\right\|_{H^{1}\left(\Omega_{i}\right)}}
$$


This modified estimate is needed later on.

Next we define the integers $K_{i}^{\prime}$ and $N_{i}^{\prime}$ as the integral parts of $\frac{K_{i}-1}{2}$ and $\frac{N_{i}-1}{2}$, respectively. For technical reasons, we introduce the modified parameter $\delta^{\prime}=$ $\left(K_{1}^{\prime}, N_{1}^{\prime}, K_{2}^{\prime}, N_{2}^{\prime}\right)$.

LEMMA 4.4. For any continuous function $t_{i}$, the following error estimate holds for any $g_{i}$ in $H^{-1}\left(\Omega_{i}\right)$ and any continuous function $\lambda_{i}$ in $H_{00}^{\frac{1}{2}}(\Gamma)$ such that $\mathcal{L}_{i}\left(t_{i}\right)\left(g_{i}, \lambda_{i}\right)$ belongs to $H^{s}\left(\Omega_{i}\right), s>1$,

$$
\begin{aligned}
& \left\|\left(\mathcal{L}_{i}-\mathcal{L}_{i \delta}\right)\left(t_{i}\right)\left(g_{i}, \lambda_{i}\right)\right\|_{H^{1}\left(\Omega_{i}\right)} \\
& \leq c \sup \left\{h_{i}^{-\frac{1}{2}}, \frac{K_{i}}{N_{i}} h_{i}^{\frac{1}{2}}\right\}\left(\left(K_{i}^{1-s}+h_{i}^{s-1} N_{i}^{1-s}\right) h_{i}^{\frac{1}{2}-s}\left\|\mathcal{L}_{i}\left(t_{i}\right)\left(g_{i}, \lambda_{i}\right)\right\|_{H^{s}\left(\Omega_{i}\right)}\right. \\
& +\left\|\lambda_{i}-\Pi_{i \delta}^{\Gamma} \lambda_{i}\right\|_{H_{00}(\Gamma)}^{\frac{1}{2}(\Gamma)} \\
& \left.+\inf _{\gamma_{i \delta^{\prime}} \in \mathbb{P}_{K_{i}^{\prime}, N_{i}^{\prime}}\left(\Omega_{i}\right)}\left\|\gamma_{i}\left(t_{i}\right)-\gamma_{i \delta^{\prime}}\right\|_{L^{\infty}\left(\Omega_{i}\right)}\left\|\mathcal{L}_{i}\left(t_{i}\right)\left(g_{i}, \lambda_{i}\right)\right\|_{H^{1}\left(\Omega_{i}\right)}\right) .
\end{aligned}
$$

Proof. We set $k_{i}=\mathcal{L}_{i}\left(t_{i}\right)\left(g_{i}, \lambda_{i}\right), k_{i \delta}=\mathcal{L}_{i \delta}\left(t_{i}\right)\left(g_{i}, \lambda_{i}\right)$. The proof is performed in several steps.

(1) We introduce an approximation $\lambda_{i \delta^{\prime}}$ of $\lambda_{i}$ in $\mathbb{P}_{K_{i}^{\prime}}(\Gamma)$ which vanishes on $\partial \Gamma$ and we take $k_{i}^{\prime}=k_{i}-R_{i}\left(\lambda_{i}-\lambda_{i \delta^{\prime}}\right)$. It follows from (4.4) that

$$
\left\|k_{i}-k_{i}^{\prime}\right\|_{H^{1}\left(\Omega_{i}\right)} \leq c h_{i}^{-\frac{1}{2}}\left\|\lambda_{i}-\lambda_{i \delta^{\prime}}\right\|_{H_{00}^{\frac{1}{2}}(\Gamma)}
$$

and also that

$$
\left\|k_{i}^{\prime}\right\|_{H^{s}\left(\Omega_{i}\right)} \leq\left\|k_{i}\right\|_{H^{s}\left(\Omega_{i}\right)}+c h_{i}^{\frac{1}{2}-s}\left(\left\|\lambda_{i}\right\|_{H^{s-\frac{1}{2}}(\Gamma)}+\left\|\lambda_{i \delta^{\prime}}\right\|_{H^{s-\frac{1}{2}(\Gamma)}}\right) .
$$

Next we set $k_{i \delta}^{\prime}=\mathcal{L}_{i \delta}\left(t_{i}\right)\left(g_{i}, \lambda_{i \delta^{\prime}}\right)$ and we deduce from Lemma 4.2 that

$$
\left\|k_{i \delta}-k_{i \delta}^{\prime}\right\|_{H^{1}\left(\Omega_{i}\right)} \leq c \sup \left\{h_{i}^{-\frac{1}{2}}, \frac{K_{i}}{N_{i}} h_{i}^{\frac{1}{2}}\right\}\left(\left\|\lambda_{i}-\lambda_{i \delta^{\prime}}\right\|_{H_{00}^{\frac{1}{2}(\Gamma)}}+\left\|\lambda_{i}-\Pi_{i \delta}^{\Gamma} \lambda_{i}\right\|_{H_{00}^{\frac{1}{2}}(\Gamma)}\right) .
$$

Thanks to the triangle inequality

$$
\left\|k_{i}-k_{i \delta}\right\|_{H^{1}\left(\Omega_{i}\right)} \leq\left\|k_{i}-k_{i}^{\prime}\right\|_{H^{1}\left(\Omega_{i}\right)}+\left\|k_{i}^{\prime}-k_{i \delta}^{\prime}\right\|_{H^{1}\left(\Omega_{i}\right)}+\left\|k_{i \delta}-k_{i \delta}^{\prime}\right\|_{H^{1}\left(\Omega_{i}\right)},
$$

it remains to estimate $\left\|k_{i}^{\prime}-k_{i \delta}^{\prime}\right\|_{H^{1}\left(\Omega_{i}\right)}$.

(2) The functions $k_{i}^{0}=k_{i}^{\prime}-R_{i \delta^{\prime}} \lambda_{i \delta^{\prime}}$ and $k_{i \delta}^{0}=k_{i \delta}^{\prime}-\mathcal{I}_{i \delta^{\prime}} R_{i \delta^{\prime}} \lambda_{i \delta^{\prime}}$ belong to $H_{0}^{1}\left(\Omega_{i}\right)$ and $Y_{i \delta}$, respectively, and satisfy

$$
\begin{aligned}
& \forall \varphi_{i} \in H_{0}^{1}\left(\Omega_{i}\right), \\
& c_{i}\left(t_{i} ; k_{i}^{0}, \varphi_{i}\right)=\int_{\Omega_{i}} g_{i} \varphi_{i} d \boldsymbol{x}-c_{i}\left(t_{i} ; R_{i \delta^{\prime}} \lambda_{i \delta^{\prime}}, \varphi_{i}\right)-c_{i}\left(t_{i} ; R_{i}\left(\lambda_{i}-\lambda_{i \delta^{\prime}}\right), \varphi_{i}\right), \\
& \forall \varphi_{i \delta} \in Y_{i \delta}, \quad c_{i \delta}\left(t_{i} ; k_{i \delta}^{0}, \varphi_{i \delta}\right)=\int_{\Omega_{i}} g_{i} \varphi_{i \delta} d \boldsymbol{x}-c_{i \delta}\left(t_{i} ; \mathcal{I}_{i \delta^{\prime}} R_{i \delta^{\prime}} \lambda_{i \delta^{\prime}}, \varphi_{i \delta}\right) .
\end{aligned}
$$

So, denoting by $\varphi_{i \delta^{\prime}}^{0}$ the orthogonal projection of $k_{i}^{0}$ onto $Y_{i \delta^{\prime}}$ for the norm of $H_{0}^{1}\left(\Omega_{i}\right)$ and adding the difference of these equations, we deduce from the ellipticity property (4.3) that

$$
\left\|k_{i \delta}^{0}-\varphi_{i \delta^{\prime}}^{0}\right\|_{H^{1}\left(\Omega_{i}\right)}^{2} \leq c c_{i \delta}\left(t_{i} ; k_{i \delta}^{0}-\varphi_{i \delta^{\prime}}^{0}, k_{i \delta}^{0}-\varphi_{i \delta^{\prime}}^{0}\right)
$$




$$
\begin{aligned}
\leq c & \left(c_{i}\left(t_{i} ; k_{i}^{0}-\varphi_{i \delta^{\prime}}^{0}, k_{i \delta}^{0}-\varphi_{i \delta^{\prime}}^{0}\right)+c_{i}\left(t_{i} ; R_{i}\left(\lambda_{i}-\lambda_{i \delta^{\prime}}\right), k_{i \delta}^{0}-\varphi_{i \delta^{\prime}}^{0}\right)\right. \\
& +c_{i}\left(t_{i} ;\left(\operatorname{Id}-\mathcal{I}_{i \delta^{\prime}}\right) R_{i \delta^{\prime}} \lambda_{i \delta^{\prime}}, k_{i \delta}^{0}-\varphi_{i \delta^{\prime}}^{0}\right) \\
& \left.\quad+\left(c_{i}-c_{i \delta}\right)\left(t_{i} ; \varphi_{i \delta^{\prime}}^{0}, k_{i \delta}^{0}-\varphi_{i \delta^{\prime}}^{0}\right)+\left(c_{i}-c_{i \delta}\right)\left(t_{i} ; \mathcal{I}_{i \delta^{\prime}} R_{i \delta^{\prime}} \lambda_{i \delta^{\prime}}, k_{i \delta}^{0}-\varphi_{i \delta^{\prime}}^{0}\right)\right)
\end{aligned}
$$

Thanks to a triangle inequality, this yields

$$
\begin{aligned}
& \left\|k_{i}^{\prime}-k_{i \delta}^{\prime}\right\|_{H^{1}\left(\Omega_{i}\right)} \\
& \leq c\left(\left\|k_{i}^{0}-\varphi_{i \delta^{\prime}}^{0}\right\|_{H^{1}\left(\Omega_{i}\right)}+c h_{i}^{-\frac{1}{2}}\left\|\lambda_{i}-\lambda_{i \delta^{\prime}}\right\|_{H_{00}^{\frac{1}{2}}(\Gamma)}+\left\|\left(\mathrm{Id}-\mathcal{I}_{i \delta^{\prime}}\right) R_{i \delta^{\prime}} \lambda_{i \delta^{\prime}}\right\|_{H^{1}\left(\Omega_{i}\right)}\right. \\
& \quad+\sup _{\chi_{i \delta} \in Y_{i \delta}} \frac{\int_{\Omega_{i}} \gamma_{i}\left(t_{i}\right) \nabla\left(\varphi_{i \delta^{\prime}}^{0}+\mathcal{I}_{i \delta^{\prime}} R_{i \delta^{\prime}} \lambda_{i \delta^{\prime}}\right) \cdot \nabla \chi_{i \delta} d \boldsymbol{x}-c_{i \delta}\left(t_{i} ; \varphi_{i \delta^{\prime}}^{0}+\mathcal{I}_{i \delta^{\prime}} R_{i \delta^{\prime}} \lambda_{i \delta^{\prime}}, \chi_{i \delta}\right)}{\left.\left\|\chi_{i \delta}\right\|_{H^{1}\left(\Omega_{i}\right)}\right) .}
\end{aligned}
$$

(3) In order to evaluate the last term, we observe that, for any $\chi_{i \delta}$ in $Y_{\delta}$, any $\psi_{i \delta^{\prime}}$ in $\mathbb{P}_{K_{i}^{\prime}, N_{i}^{\prime}}\left(\Omega_{i}\right)$, and any $\gamma_{i \delta^{\prime}}$ in $\mathbb{P}_{K_{i}^{\prime}, N_{i}^{\prime}}\left(\Omega_{i}\right)$,

$$
\int_{\Omega_{i}} \gamma_{i \delta^{\prime}} \nabla \psi_{i \delta^{\prime}} \cdot \nabla \chi_{i \delta} d \boldsymbol{x}=\left(\gamma_{i \delta^{\prime}} \nabla \psi_{i \delta^{\prime}}, \nabla \chi_{i \delta}\right)_{i \delta}
$$

Adding and subtracting this quantity and using the continuity property (4.2) leads to, for any $\chi_{i \delta}$ in $Y_{i \delta}$,

$$
\begin{aligned}
\int_{\Omega_{i}} \gamma_{i}\left(t_{i}\right) \nabla\left(\varphi_{i \delta^{\prime}}^{0}+\mathcal{I}_{i \delta^{\prime}} R_{i \delta^{\prime}} \lambda_{i \delta^{\prime}}\right) \cdot \nabla \chi_{i \delta} d \boldsymbol{x}-c_{i \delta}\left(t_{i} ; \varphi_{i \delta^{\prime}}^{0}+\mathcal{I}_{i \delta^{\prime}} R_{i \delta^{\prime}} \lambda_{i \delta^{\prime}}, \chi_{i \delta}\right) \\
\quad \leq c\left\|\gamma_{i}\left(t_{i}\right)-\gamma_{i \delta^{\prime}}\right\|_{L^{\infty}\left(\Omega_{i}\right)}\left\|\varphi_{i \delta^{\prime}}^{0}+\mathcal{I}_{i \delta^{\prime}} R_{i \delta^{\prime}} \lambda_{i \delta^{\prime}}\right\|_{H^{1}\left(\Omega_{i}\right)}\left\|\chi_{i \delta}\right\|_{H^{1}\left(\Omega_{i}\right)} .
\end{aligned}
$$

Moreover, it follows from the definition of $\varphi_{i \delta^{\prime}}^{0}$ that

$$
\begin{aligned}
\left\|\varphi_{i \delta^{\prime}}^{0}+\mathcal{I}_{i \delta^{\prime}} R_{i \delta^{\prime}} \lambda_{i \delta^{\prime}}\right\|_{H^{1}\left(\Omega_{i}\right)} & \leq\left\|k_{i}^{0}\right\|_{H^{1}\left(\Omega_{i}\right)}+\left\|\mathcal{I}_{i \delta^{\prime}} R_{i \delta^{\prime}} \lambda_{i \delta^{\prime}}\right\|_{H^{1}\left(\Omega_{i}\right)} \\
& \leq\left\|k_{i}^{\prime}\right\|_{H^{1}\left(\Omega_{i}\right)}+\left\|R_{i \delta^{\prime}} \lambda_{i \delta^{\prime}}\right\|_{H^{1}\left(\Omega_{i}\right)}+\left\|\mathcal{I}_{i \delta^{\prime}} R_{i \delta^{\prime}} \lambda_{i \delta^{\prime}}\right\|_{H^{1}\left(\Omega_{i}\right)} .
\end{aligned}
$$

Thanks to (4.5) and (4.6), we obtain

$$
\left\|\varphi_{i \delta^{\prime}}^{0}+\mathcal{I}_{i \delta^{\prime}} R_{i \delta^{\prime}} \lambda_{i \delta^{\prime}}\right\|_{H^{1}\left(\Omega_{i}\right)} \leq\left\|k_{i}^{\prime}\right\|_{H^{1}\left(\Omega_{i}\right)}+c \sup \left\{h_{i}^{-\frac{1}{2}}, \frac{K_{i}}{N_{i}} h_{i}^{\frac{1}{2}}\right\}\left\|\lambda_{i \delta^{\prime}}\right\|_{H_{00}^{\frac{1}{2}}(\Gamma)} .
$$

(4) To conclude, we note that the trace $\lambda_{i}$ of $k_{i}$ belongs to $H^{s-\frac{1}{2}}(\Gamma)$ and choose the polynomial $\lambda_{i \delta^{\prime}}$ such that (see [7, Thm. 7.4])

$$
\left\|\lambda_{i}-\lambda_{i \delta^{\prime}}\right\|_{H_{00}^{\frac{1}{2}(\Gamma)}} \leq c K_{i}^{1-s}\left\|\lambda_{i}\right\|_{H^{s-\frac{1}{2}}(\Gamma)}, \quad\left\|\lambda_{i \delta^{\prime}}\right\|_{H^{s-\frac{1}{2}(\Gamma)}} \leq c\left\|\lambda_{i}\right\|_{H^{s-\frac{1}{2}}(\Gamma)} .
$$

Next it can be observed that, for any polynomial $r_{i \delta^{\prime}}$ in $\mathbb{P}_{K_{i}^{\prime}, N_{i}^{\prime}}\left(\Omega_{i}\right)$,

$$
\left\|\left(\operatorname{Id}-\mathcal{I}_{i \delta^{\prime}}\right) R_{i \delta^{\prime}} \lambda_{i \delta^{\prime}}\right\|_{H^{1}\left(\Omega_{i}\right)}=\left\|\left(\operatorname{Id}-\mathcal{I}_{i \delta^{\prime}}\right)\left(R_{i \delta^{\prime}} \lambda_{i \delta^{\prime}}-r_{i \delta^{\prime}}\right)\right\|_{H^{1}\left(\Omega_{i}\right)} .
$$

Using the stability properties of the operator $\mathcal{I}_{i \delta^{\prime}}$ on polynomials (see [7, Forms. (13.27) and (13.28)]) and taking $r_{i \delta^{\prime}}$ equal to the orthogonal projection of $R_{i \delta^{\prime}} \lambda_{i \delta^{\prime}}$ in $H^{1}\left(\Omega_{i}\right)$ yields

$\left\|\left(\operatorname{Id}-\mathcal{I}_{i \delta^{\prime}}\right) R_{i \delta^{\prime}} \lambda_{i \delta^{\prime}}\right\|_{H^{1}\left(\Omega_{i}\right)} \leq c \sup \left\{h_{i}^{-\frac{1}{2}}, \frac{K_{i}}{N_{i}} h_{i}^{\frac{1}{2}}\right\}\left(K_{i}^{1-s}+h_{i}^{s-1} N_{i}^{1-s}\right)\left\|R_{i \delta^{\prime}} \lambda_{i \delta^{\prime}}\right\|_{H^{s}\left(\Omega_{i}\right)}$, 
whence, from (4.5),

$$
\left\|\left(\operatorname{Id}-\mathcal{I}_{i \delta^{\prime}}\right) R_{i \delta^{\prime}} \lambda_{i \delta^{\prime}}\right\|_{H^{1}\left(\Omega_{i}\right)} \leq c \sup \left\{h_{i}^{-\frac{1}{2}}, \frac{K_{i}}{N_{i}} h_{i}^{\frac{1}{2}}\right\}\left(K_{i}^{1-s}+h_{i}^{s-1} N_{i}^{1-s}\right) h^{\frac{1}{2}-s}\left\|\lambda_{i \delta^{\prime}}\right\|_{H^{s-\frac{1}{2}}(\Gamma)} .
$$

Finally, using the previous estimates also yields

$$
\left\|k_{i}^{0}-\varphi_{i \delta^{\prime}}^{0}\right\|_{H^{1}\left(\Omega_{i}\right)} \leq c\left(K_{i}^{1-s}+h_{i}^{s-1} N_{i}^{1-s}\right)\left(\left\|k_{i}^{\prime}\right\|_{H^{s}\left(\Omega_{i}\right)}+h_{i}^{\frac{1}{2}-s}\left\|\lambda_{i \delta^{\prime}}\right\|_{H^{s-\frac{1}{2}(\Gamma)}}\right) .
$$

To conclude, we observe that $\left\|\lambda_{i}\right\|_{H^{s-\frac{1}{2}(\Gamma)}}$ is bounded by a constant $\left\|k_{i}\right\|_{H^{s}\left(\Omega_{i}\right)}$. This ends the proof.

Remark 4.5. The following estimate can be derived by combining [7, Thm. 7.4] with a Gagliardo-Nirenberg inequality: if the function $\gamma_{i}$ is of class $\mathrm{C}^{m}$ with bounded derivatives of order $\leq m$ and if the function $t_{i}$ belongs to $H^{s}\left(\Omega_{i}\right), \frac{d}{2}<s \leq m$,

$$
\inf _{\gamma_{i \delta^{\prime}} \in \mathbb{P}_{K_{i}, N_{i}}\left(\Omega_{i}\right)}\left\|\gamma_{i}\left(t_{i}\right)-\gamma_{i \delta^{\prime}}\right\|_{L^{\infty}\left(\Omega_{i}\right)} \leq c\left(K_{i}^{\frac{d}{2}-s}+h_{i}^{s-\frac{d}{2}} N_{i}^{\frac{d}{2}-s}\right)\left\|t_{i}\right\|_{H^{s}\left(\Omega_{i}\right)} .
$$

Moreover, a more sophisticated argument, using the full regularity of $\mathcal{L}_{i}\left(t_{i}\right)\left(g_{i}, \lambda_{i}\right)$ allows us to replace when $s$ is $>\frac{d}{2}$ the last term in (4.9) by the better estimate

$$
c\left(K_{i}^{1-s}+h_{i}^{s-1} N_{i}^{1-s}\right)\left\|t_{i}\right\|_{H^{s}\left(\Omega_{i}\right)}\left\|\mathcal{L}_{i}\left(t_{i}\right)\left(g_{i}, \lambda_{i}\right)\right\|_{H^{s}\left(\Omega_{i}\right)} .
$$

Remark 4.6. If the function $\gamma_{i}$ is differentiable with bounded derivative and if the function $t_{i}$ belongs to $H^{r}\left(\Omega_{i}\right)$ for $r>1$, the Aubin-Nitsche duality argument [7, Thm. 15.4] leads to the improved estimate

$$
\begin{aligned}
&\left\|\left(\mathcal{L}_{i}-\mathcal{L}_{i \delta}\right)\left(t_{i}\right)\left(g_{i}, \lambda_{i}\right)\right\|_{H^{1-\varepsilon}\left(\Omega_{i}\right)} \\
& \leq c\left(\left(K_{i}^{-\varepsilon}+h_{i}^{\varepsilon} N_{i}^{-\varepsilon}\right)\left\|\left(\mathcal{L}_{i}-\mathcal{L}_{i \delta}\right)\left(t_{i}\right)\left(g_{i}, \lambda_{i}\right)\right\|_{H^{1}\left(\Omega_{i}\right)}\right. \\
&\left.+\inf _{\gamma_{i \delta^{\prime}} \in \mathbb{P}_{K_{i}^{\prime}, N_{i}^{\prime}}\left(\Omega_{i}\right)}\left\|\gamma_{i}\left(t_{i}\right)-\gamma_{i \delta^{\prime}}\right\|_{L^{\infty}\left(\Omega_{i}\right)}\left\|\mathcal{L}_{i}\left(t_{i}\right)\left(g_{i}, \lambda_{i}\right)\right\|_{H^{1}\left(\Omega_{i}\right)}\right) .
\end{aligned}
$$

Finally, we investigate the dependency of $\mathcal{L}_{i \delta}\left(t_{i}\right)\left(g_{i}, \lambda_{i}\right)$ with respect to $t_{i}$.

Lemma 4.7. For any continuous functions $t_{i}$ and $t_{i}^{\prime}$, the following stability property holds for any $g_{i}$ in $H^{-1}\left(\Omega_{i}\right)$ and any continuous function $\lambda_{i}$ on $\Gamma$ :

$$
\begin{aligned}
\| \mathcal{L}_{i \delta}\left(t_{i}\right)\left(g_{i}, \lambda_{i}\right)-\mathcal{L}_{i \delta}\left(t_{i}^{\prime}\right) & \left(g_{i}, \lambda_{i}\right) \|_{H^{1}\left(\Omega_{i}\right)} \\
& \leq c\left\|\gamma_{i}\left(t_{i}\right)-\gamma_{i}\left(t_{i}^{\prime}\right)\right\|_{L^{\infty}\left(\Omega_{i}\right)}\left\|\mathcal{L}_{i \delta}\left(t_{i}\right)\left(g_{i}, \lambda_{i}\right)\right\|_{H^{1}\left(\Omega_{i}\right)} .
\end{aligned}
$$

Proof. Setting $k_{i \delta}=\mathcal{L}_{i \delta}\left(t_{i}\right)\left(g_{i}, \lambda_{i}\right)$ and $k_{i \delta}^{\prime}=\mathcal{L}_{i \delta}\left(t_{i}^{\prime}\right)\left(g_{i}, \lambda_{i}\right)$, we observe that the function $k_{i \delta}-k_{i \delta}^{\prime}$ belongs to $Y_{i \delta}$ and satisfies

$$
\forall \varphi_{i \delta} \in Y_{i \delta}, \quad\left(\gamma_{i}\left(t_{i}\right) \nabla k_{i \delta}, \nabla \varphi_{i \delta}\right)_{i \delta}=\left(\gamma_{i}\left(t_{i}^{\prime}\right) \nabla k_{i \delta}^{\prime}, \nabla \varphi_{i \delta}\right)_{i \delta},
$$

whence

$$
\begin{aligned}
c_{i \delta}\left(t_{i}^{\prime} ; k_{i \delta}-k_{i \delta}^{\prime}, k_{i \delta}-k_{i \delta}^{\prime}\right) & =\left(\gamma_{i}\left(t_{i}^{\prime}\right)\left(\nabla k_{i \delta}-\nabla k_{i \delta}^{\prime}\right),\left(\nabla k_{i \delta}-\nabla k_{i \delta}^{\prime}\right)\right)_{i \delta} \\
& =-\left(\left(\gamma_{i}\left(t_{i}\right)-\gamma_{i}\left(t_{i}^{\prime}\right)\right) \nabla k_{i \delta},\left(\nabla k_{i \delta}-\nabla k_{i \delta}^{\prime}\right)\right)_{i \delta} .
\end{aligned}
$$

So the desired estimated follows from the properties of $c_{i \delta}(\cdot ; \cdot, \cdot)$; see Lemma 4.1. 
The discrete Stokes operator. We now present similar properties for the Stokes operator $\mathcal{S}_{i \delta}$ defined by (3.14); however, we skip the proofs except for the error estimates.

LEMma 4.8. For any continuous function $t_{i}$, the form $a_{i \delta}\left(t_{i} ; \cdot, \cdot\right)$ satisfies the following properties of continuity:

$$
\forall \boldsymbol{u}_{i \delta} \in X_{i \delta}, \forall \boldsymbol{v}_{i \delta} \in X_{i \delta}, \quad a_{i \delta}\left(t_{i} ; \boldsymbol{u}_{i \delta}, \boldsymbol{v}_{i \delta}\right) \leq c\left\|\boldsymbol{u}_{i \delta}\right\|_{H^{1}\left(\Omega_{i}\right)^{d}}\left\|\boldsymbol{v}_{i \delta}\right\|_{H^{1}\left(\Omega_{i}\right)^{d}},
$$

and of ellipticity:

$$
\forall \boldsymbol{v}_{i \delta} \in X_{i \delta}, \quad a_{i \delta}\left(t_{i} ; \boldsymbol{v}_{i \delta}, \boldsymbol{v}_{i \delta}\right) \geq c\left\|\boldsymbol{v}_{i \delta}\right\|_{H^{1}\left(\Omega_{i}\right)^{d}}^{2} .
$$

LEMma 4.9. For any continuous function $t_{i}$, the following stability property holds for any $\boldsymbol{g}_{i}$ in $L^{2}\left(\Omega_{i}\right)^{d}$ and any $\boldsymbol{\lambda}_{i}$ in the dual space of $H_{00}^{\frac{1}{2}}(\Gamma)^{d}$ :

$$
\left\|\mathcal{S}_{i \delta}\left(t_{i}\right)\left(\boldsymbol{g}_{i}, \boldsymbol{\lambda}_{i}\right)\right\|_{H^{1}\left(\Omega_{i}\right)^{d}} \leq c\left(\left\|\boldsymbol{g}_{i}\right\|_{L^{2}\left(\Omega_{i}\right)^{d}}+\left\|\boldsymbol{\lambda}_{i}\right\|_{H_{00}^{\frac{1}{2}}(\Gamma)^{\prime} d}\right) .
$$

Remark 4.10. As for the Laplace operator, the norms of $\boldsymbol{g}_{i}$ and $\boldsymbol{\lambda}_{i}$ in the righthand side can be replaced, respectively, by the dual norms of $X_{i \delta}$ (when provided with the norm $\left.\|\cdot\|_{H^{1}\left(\Omega_{i}\right)^{d}}\right)$ and of $\mathbb{P}_{K_{i}}(\Gamma)^{d} \cap H_{00}^{\frac{1}{2}}(\Gamma)^{d}$ (provided with the norm $\|\cdot\|_{H_{00}^{\frac{1}{2}}(\Gamma)^{d}}$ ).

However, the proof of the convergence estimate is slightly different (but simpler).

LEMma 4.11. For any continuous function $t_{i}$, the following error estimate holds for any $\boldsymbol{g}_{i}$ in $L^{2}\left(\Omega_{i}\right)^{d}$ and any $\boldsymbol{\lambda}_{i}$ in the dual space of $H_{00}^{\frac{1}{2}}(\Gamma)^{d}$ :

$$
\begin{aligned}
&\left\|\left(\mathcal{S}_{i}-\mathcal{S}_{i \delta}\right)\left(t_{i}\right)\left(\boldsymbol{g}_{i}, \boldsymbol{\lambda}_{i}\right)\right\|_{H^{1}\left(\Omega_{i}\right)^{d}} \\
& \leq c \inf _{\boldsymbol{w}_{i \delta} \in X_{i \delta^{\prime}} \cap V_{i}}\left(\left\|\mathcal{S}_{i}\left(t_{i}\right)\left(\boldsymbol{g}_{i}, \boldsymbol{\lambda}_{i}\right)-\boldsymbol{w}_{i \delta}\right\|_{H^{1}\left(\Omega_{i}\right)^{d}}\right. \\
&\left.+\inf _{\alpha_{i \delta^{\prime}} \in \mathbb{P}_{K_{i}^{\prime}, N_{i}^{\prime}}\left(\Omega_{i}\right)}\left\|\alpha_{i}\left(t_{i}\right)-\alpha_{i \delta^{\prime}}\right\|_{L^{\infty}\left(\Omega_{i}\right)}\left\|\boldsymbol{w}_{i \delta}\right\|_{H^{1}\left(\Omega_{i}\right)^{d}}\right) .
\end{aligned}
$$

Proof. Setting $\boldsymbol{u}_{i}=\mathcal{S}_{i}\left(t_{i}\right)\left(\boldsymbol{g}_{i}, \boldsymbol{\lambda}_{i}\right)$ and $\boldsymbol{u}_{i \delta}=\mathcal{S}_{i \delta}\left(t_{i}\right)\left(\boldsymbol{g}_{i}, \boldsymbol{\lambda}_{i}\right)$, we derive from (4.16) that, for any $\boldsymbol{w}_{i \delta^{\prime}}$ in $X_{i \delta^{\prime}} \cap V_{i}$,

$$
\left\|\boldsymbol{u}_{i \delta}-\boldsymbol{w}_{i \delta^{\prime}}\right\|_{H^{1}\left(\Omega_{i}\right)^{d}}^{2} \leq c a_{i \delta}\left(t_{i} ; \boldsymbol{u}_{i \delta}-\boldsymbol{w}_{i \delta^{\prime}}, \boldsymbol{u}_{i \delta}-\boldsymbol{w}_{i \delta^{\prime}}\right) .
$$

Using (2.14) (in variational form) and (3.14), we derive

$$
\left\|\boldsymbol{u}_{i \delta}-\boldsymbol{w}_{i \delta^{\prime}}\right\|_{H^{1}\left(\Omega_{i}\right)^{d}}^{2} \leq c\left(a_{i}\left(t_{i} ; \boldsymbol{u}_{i}, \boldsymbol{u}_{i \delta}-\boldsymbol{w}_{i \delta^{\prime}}\right)-a_{i \delta}\left(t_{i} ; \boldsymbol{w}_{i \delta^{\prime}}, \boldsymbol{u}_{i \delta}-\boldsymbol{w}_{i \delta^{\prime}}\right)\right) .
$$

Next we deduce from the exactness of the quadrature formula that, for any $\alpha_{i \delta^{\prime}}$ in $\mathbb{P}_{K_{i}^{\prime}, N_{i}^{\prime}}\left(\Omega_{i}\right)$

$$
\int_{\Omega_{i}} \alpha_{i \delta^{\prime}} \nabla \boldsymbol{w}_{i \delta^{\prime}} \cdot \nabla\left(\boldsymbol{u}_{i \delta}-\boldsymbol{w}_{i \delta^{\prime}}\right) d \boldsymbol{x}=\left(\alpha_{i \delta^{\prime}} \nabla \boldsymbol{w}_{i \delta^{\prime}}, \nabla\left(\boldsymbol{u}_{i \delta}-\boldsymbol{w}_{i \delta^{\prime}}\right)\right)_{i \delta} .
$$

Adding and subtracting this quantity yield

$$
\begin{aligned}
\| \boldsymbol{u}_{i \delta} & -\boldsymbol{w}_{i \delta^{\prime}} \|_{H^{1}\left(\Omega_{i}\right)^{d}}^{2} \leq c\left(a_{i}\left(t_{i} ; \boldsymbol{u}_{i}-\boldsymbol{w}_{i \delta^{\prime}}, \boldsymbol{u}_{i \delta}-\boldsymbol{w}_{i \delta^{\prime}}\right)\right. \\
& +\int_{\Omega_{i}}\left(\alpha_{i}\left(t_{i}\right)-\alpha_{i \delta^{\prime}}\right) \nabla \boldsymbol{w}_{i \delta^{\prime}} \cdot \nabla\left(\boldsymbol{u}_{i \delta}-\boldsymbol{w}_{i \delta^{\prime}}\right) d \boldsymbol{x} \\
& \left.-\left(\left(\alpha_{i}\left(t_{i}\right)-\alpha_{i \delta^{\prime}}\right) \nabla \boldsymbol{w}_{i \delta^{\prime}}, \nabla\left(\boldsymbol{u}_{i \delta}-\boldsymbol{w}_{i \delta^{\prime}}\right)\right)_{i \delta}\right) .
\end{aligned}
$$


So the desired estimate follows from the continuity property (4.15), together with a triangle inequality.

Remark 4.12. In dimension $d=2$, it is easy to evaluate the distance of a function $\boldsymbol{u}_{i}$ in $V_{i}$ to $X_{i \delta} \cap V_{i}$ by introducing the stream function $\psi_{i}$ such that $\boldsymbol{u}_{i}=\operatorname{curl} \psi_{i}$. Indeed, the functions curl $\psi_{i \delta}$, where $\psi_{i \delta}$ belongs to $\mathbb{P}_{K_{i}, N_{i}}\left(\Omega_{i}\right)$, satisfies the desired boundary conditions and approximates $\psi_{i}$ in $H^{2}\left(\Omega_{i}\right)$, belongs to $X_{i \delta} \cap V_{i}$, and provides a good approximation of $\boldsymbol{u}_{i}$. The case of dimension $d=3$ is more complex; however, the right approximation properties have been proved in [22] for smooth functions and extended in [5] to arbitrary functions. So the general result reads as follows: for any function $\boldsymbol{u}_{i}$ in $H^{s}\left(\Omega_{i}\right)^{d} \cap V_{i}, s \geq 1$,

$$
\inf _{\boldsymbol{w}_{i \delta} \in X_{i \delta} \cap V_{i}}\left\|\mathcal{S}_{i}\left(t_{i}\right)\left(\boldsymbol{g}_{i}, \boldsymbol{\lambda}_{i}\right)-\boldsymbol{w}_{i \delta}\right\|_{H^{1}\left(\Omega_{i}\right)^{d}} \leq c\left(K_{i}^{1-s}+h_{i}^{s-1} N_{i}^{1-s}\right)\left\|\boldsymbol{u}_{i}\right\|_{H^{s}\left(\Omega_{i}\right)^{d}} .
$$

Note also that the last term in (4.18) can be bounded analogously to (4.11) or (4.12).

LEMma 4.13. For any continuous functions $t_{i}$ and $t_{i}^{\prime}$, the following stability property holds for any $\boldsymbol{g}_{i}$ in $L^{2}\left(\Omega_{i}\right)^{d}$ and any $\boldsymbol{\lambda}_{i}$ in the dual space of $H_{00}^{\frac{1}{2}}(\Gamma)^{d}$ :

$$
\begin{aligned}
\| \mathcal{S}_{i \delta}\left(t_{i}\right)\left(\boldsymbol{g}_{i}, \boldsymbol{\lambda}_{i}\right)-\mathcal{S}_{i \delta}\left(t_{i}^{\prime}\right) & \left(\boldsymbol{g}_{i}, \boldsymbol{\lambda}_{i}\right) \|_{H^{1}\left(\Omega_{i}\right)^{d}} \\
& \leq c\left\|\alpha_{i}\left(t_{i}\right)-\alpha_{i}\left(t_{i}^{\prime}\right)\right\|_{L^{\infty}\left(\Omega_{i}\right)}\left\|\mathcal{S}_{i \delta}\left(t_{i}\right)\left(\boldsymbol{g}_{i}, \boldsymbol{\lambda}_{i}\right)\right\|_{H^{1}\left(\Omega_{i}\right)^{d}} .
\end{aligned}
$$

5. Numerical analysis of the discrete problem. The aim of this section is to prove that, if Hypotheses 2.2 and 2.4 hold, problem (3.4)-(3.5) has a unique solution in a neighborhood of $\left(U_{1}^{*}, U_{2}^{*}\right)$ and that this solution converges to $\left(U_{1}^{*}, U_{2}^{*}\right)$. We also derive optimal error estimates. To this aim, we check the assumptions of the theorem of Brezzi, Rappaz, and Raviart [10] in Propositions 5.5 to 5.7.

From now on, we denote by $\mathcal{X}_{i \delta}, i=1$ and 2 , the space $X_{i \delta} \times \mathbb{P}_{K_{i}, N_{i}}\left(\Omega_{i}\right)$, provided with the norm of $\mathcal{X}_{i}$, and by $\mathcal{X}_{\delta}$ the product $\mathcal{X}_{1 \delta} \times \mathcal{X}_{2 \delta}$.

In view of (4.6), (4.9), and (4.19), for instance, we decide to take the $N_{i}, i=$ 1 and 2 , such that, for a fixed constant $\kappa$,

$$
\kappa h_{i} K_{i} \leq N_{i}<\kappa h_{i} K_{i}+1
$$

and we do not any longer take into account the dependency of the constants with respect to the $h_{i}$. We also choose an approximation $\left(U_{1 \delta^{\prime}}^{*}, U_{2 \delta^{\prime}}^{*}\right)$, with $U_{i \delta^{\prime}}^{*}=\left(\boldsymbol{u}_{i \delta^{\prime}}^{*}, k_{i \delta^{\prime}}^{*}\right)$, of the solution $\left(U_{1}^{*}, U_{2}^{*}\right)$ in $\prod_{i=1}^{2}\left(X_{i \delta^{\prime}} \times P_{K_{i}^{\prime}, N_{i}^{\prime}}\left(\Omega_{i}\right)\right)$ which satisfies the following approximation properties for $0 \leq r \leq s^{*}$, where $s^{*}$ is introduced in Hypothesis 2.2:

$$
\begin{aligned}
& \left\|\boldsymbol{u}_{i}^{*}-\boldsymbol{u}_{i \delta^{\prime}}^{*}\right\|_{H^{r}\left(\Omega_{i}\right)^{d}} \leq c K_{i}^{r-s^{*}}\left\|\boldsymbol{u}_{i}^{*}\right\|_{H^{s^{*}}\left(\Omega_{i}\right)^{d}}, \\
& \left\|k_{i}^{*}-k_{i \delta^{\prime}}^{*}\right\|_{H^{r}\left(\Omega_{i}\right)} \leq c K_{i}^{r-s^{*}}\left\|k_{i}^{*}\right\|_{H^{s^{*}}\left(\Omega_{i}\right)} .
\end{aligned}
$$

The existence of such an approximation is stated in [7, Thm. 7.4]. Finally, we assume that the functions $\alpha_{i}$ and $\gamma_{i}$ are of class $\mathrm{C}^{2}$, with bounded derivatives up to order 2 and also that the operators $\Pi_{i \delta}^{\Gamma}$ satisfy, for all $s \geq \frac{1}{2}$ (the notation $H_{\diamond}^{s-\frac{1}{2}}(\Gamma)$ is introduced in (4.4)),

$$
\forall \lambda \in H_{\diamond}^{s-\frac{1}{2}}(\Gamma), \quad\left\|\lambda-\Pi_{i \delta}^{\Gamma} \lambda\right\|_{H_{00}^{\frac{1}{2}}\left(\Omega_{i}\right)} \leq c K_{i}^{1-s}\|\lambda\|_{H_{\diamond}^{s-\frac{1}{2}}(\Gamma)} .
$$

In a first step, we must prove the analogue of Hypothesis 2.4 for the discrete operator. The proof relies on the expansion

$$
\begin{aligned}
\operatorname{Id}+D \mathcal{T}_{\delta}\left(k_{1 \delta^{\prime}}^{*}, k_{2 \delta^{\prime}}^{*}\right) \mathcal{G}_{\delta}\left(U_{1 \delta^{\prime}}^{*}, U_{2 \delta^{\prime}}^{*}\right)+\mathcal{T}_{\delta}\left(k_{1 \delta^{\prime}}^{*}, k_{2 \delta^{\prime}}^{*}\right) D \mathcal{G}_{\delta}\left(U_{1 \delta^{\prime}}^{*}, U_{2 \delta^{\prime}}^{*}\right) \\
=\operatorname{Id}+D \mathcal{T}\left(k_{1}^{*}, k_{2}^{*}\right) \mathcal{G}\left(U_{1}^{*}, U_{2}^{*}\right)+\mathcal{T}\left(k_{1}^{*}, k_{2}^{*}\right) D \mathcal{G}\left(U_{1}^{*}, U_{2}^{*}\right)+\sum_{j=1}^{4} \mathcal{O}_{j},
\end{aligned}
$$


with

$$
\begin{aligned}
& \mathcal{O}_{1}=-\left(D \mathcal{T}\left(k_{1}^{*}, k_{2}^{*}\right) \mathcal{G}\left(U_{1}^{*}, U_{2}^{*}\right)-\right.\left.D \mathcal{T}_{\delta}\left(k_{1}^{*}, k_{2}^{*}\right) \mathcal{G}\left(U_{1 \delta^{\prime}}^{*}, U_{2 \delta^{\prime}}^{*}\right)\right) \\
&-\left(\mathcal{T}-\mathcal{T}_{\delta}\right)\left(k_{1}^{*}, k_{2}^{*}\right) D \mathcal{G}\left(U_{1}^{*}, U_{2}^{*}\right), \\
& \mathcal{O}_{2}=-\left(D \mathcal{T}_{\delta}\left(k_{1}^{*}, k_{2}^{*}\right)-D \mathcal{T}_{\delta}\left(k_{1 \delta^{\prime}}^{*}, k_{2 \delta^{\prime}}^{*}\right)\right) \mathcal{G}\left(U_{1 \delta^{\prime}}^{*}, U_{2 \delta^{\prime}}^{*}\right)-\left(\mathcal{T}_{\delta}\left(k_{1}^{*}, k_{2}^{*}\right)-\mathcal{T}_{\delta}\left(k_{1 \delta^{\prime}}^{*}, k_{2 \delta^{\prime}}^{*}\right)\right) D \mathcal{G}\left(U_{1}^{*}, U_{2}^{*}\right), \\
& \mathcal{O}_{3}=-\mathcal{T}_{\delta}\left(k_{1 \delta^{\prime}}^{*}, k_{2 \delta^{\prime}}^{*}\right)\left(D \mathcal{G}\left(U_{1}^{*}, U_{2}^{*}\right)-D \mathcal{G}\left(U_{1 \delta^{\prime}}^{*}, U_{2 \delta^{\prime}}^{*}\right)\right), \\
& \mathcal{O}_{4}=-D \mathcal{T}_{\delta}\left(k_{1 \delta^{\prime}}^{*}, k_{2 \delta^{\prime}}^{*}\right)\left(\mathcal{G}\left(U_{1 \delta^{\prime}}^{*}, U_{2 \delta^{\prime}}^{*}\right)-\mathcal{G}_{\delta}\left(U_{1 \delta^{\prime}}^{*}, U_{2 \delta^{\prime}}^{*}\right)\right) \\
&-\mathcal{T}_{\delta}\left(k_{1 \delta^{\prime}}^{*}, k_{2 \delta^{\prime}}^{*}\right)\left(D \mathcal{G}\left(U_{1 \delta^{\prime}}^{*}, U_{2 \delta^{\prime}}^{*}\right)-D \mathcal{G}_{\delta}\left(U_{1 \delta^{\prime}}^{*}, U_{2 \delta^{\prime}}^{*}\right)\right) .
\end{aligned}
$$

So we now prove that each $\mathcal{O}_{j}$ tends to zero when $K_{1}$ and $K_{2}$ go to $+\infty$, in the norm of the space $\mathrm{L}\left(\mathcal{X}_{\delta}, \mathcal{X}_{\delta}\right)$ of linear mappings from $\mathcal{X}_{\delta}$ into itself. These properties are stated in the following lemmas.

Let us first observe that, for any $W=\left(W_{1}, W_{2}\right)$ in $\mathcal{X}$, with $W_{i}=\left(\boldsymbol{w}_{i}, m_{i}\right)$,

$$
D \mathcal{G}\left(U_{1}^{*}, U_{2}^{*}\right) \cdot W=\left(\begin{array}{c}
\left(\mathbf{0}, D \boldsymbol{\lambda}_{1}\left(\boldsymbol{u}_{1}^{*}, \boldsymbol{u}_{2}^{*}\right) \cdot\left(\boldsymbol{w}_{1}, \boldsymbol{w}_{2}\right)\right) \\
\left(-D g_{1}\left(k_{1}^{*}, \boldsymbol{u}_{1}^{*}\right) \cdot\left(m_{1}, \boldsymbol{w}_{1}\right), D \lambda\left(\boldsymbol{u}_{1}^{*}, \boldsymbol{u}_{2}^{*}\right) \cdot\left(\boldsymbol{w}_{1}, \boldsymbol{w}_{2}\right)\right) \\
\left(\mathbf{0}, D \boldsymbol{\lambda}_{2}\left(\boldsymbol{u}_{1}^{*}, \boldsymbol{u}_{2}^{*}\right) \cdot\left(\boldsymbol{w}_{1}, \boldsymbol{w}_{2}\right)\right) \\
\left(-D g_{2}\left(k_{2}^{*}, \boldsymbol{u}_{2}^{*}\right) \cdot\left(m_{2}, \boldsymbol{w}_{2}\right), D \lambda\left(\boldsymbol{u}_{1}^{*}, \boldsymbol{u}_{2}^{*}\right) \cdot\left(\boldsymbol{w}_{1}, \boldsymbol{w}_{2}\right)\right)
\end{array}\right)
$$

with

$$
\begin{gathered}
D \boldsymbol{\lambda}_{i}\left(\boldsymbol{u}_{1}^{*}, \boldsymbol{u}_{2}^{*}\right) \cdot\left(\boldsymbol{w}_{1}, \boldsymbol{w}_{2}\right)=\left|\boldsymbol{u}_{i}^{*}-\boldsymbol{u}_{j}^{*}\right|\left(\boldsymbol{w}_{i}-\boldsymbol{w}_{j}\right)+\frac{\left(\boldsymbol{u}_{i}^{*}-\boldsymbol{u}_{j}^{*}\right) \cdot\left(\boldsymbol{w}_{i}-\boldsymbol{w}_{j}\right)}{\left|\boldsymbol{u}_{i}^{*}-\boldsymbol{u}_{j}^{*}\right|}\left(\boldsymbol{u}_{i}^{*}-\boldsymbol{u}_{j}^{*}\right), \\
D g_{i}\left(k_{i}^{*}, \boldsymbol{u}_{i}^{*}\right) \cdot\left(m_{i}, \boldsymbol{w}_{i}\right)=2 \alpha_{i}\left(k_{i}^{*}\right) \nabla \boldsymbol{u}_{i}^{*} \cdot \nabla \boldsymbol{w}_{i}+\alpha_{i}^{\prime}\left(k_{i}^{*}\right) m_{i}\left|\nabla \boldsymbol{u}_{i}^{*}\right|^{2} \\
D \lambda\left(\boldsymbol{u}_{1}^{*}, \boldsymbol{u}_{2}^{*}\right) \cdot\left(\boldsymbol{w}_{1}, \boldsymbol{w}_{2}\right)=-2\left(\boldsymbol{u}_{1}^{*}-\boldsymbol{u}_{2}^{*}\right) \cdot\left(\boldsymbol{w}_{1}-\boldsymbol{w}_{2}\right) .
\end{gathered}
$$

Moreover, we have the following formula (which can be derived from the explicit form (2.20)-(2.21) of the linearized problem):

$$
\begin{aligned}
D \mathcal{T}\left(k_{1}^{*}, k_{2}^{*}\right) \mathcal{G}\left(U_{1}^{*}, U_{2}^{*}\right)+\mathcal{T}\left(k_{1}^{*}, k_{2}^{*}\right) & D \mathcal{G}\left(U_{1}^{*}, U_{2}^{*}\right) \\
& =\mathcal{T}\left(k_{1}^{*}, k_{2}^{*}\right)\left(D \mathcal{G}\left(U_{1}^{*}, U_{2}^{*}\right)+D \mathcal{H}\left(U_{1}^{*}, U_{2}^{*}\right)\right),
\end{aligned}
$$

with

$$
D \mathcal{H}\left(U_{1}^{*}, U_{2}^{*}\right) \cdot\left(m_{1}, m_{2}\right)=\left(\begin{array}{c}
\left(-\operatorname{div}\left(\alpha_{1}^{\prime}\left(k_{1}^{*}\right) m_{1} \nabla \boldsymbol{u}_{1}^{*}\right), \mathbf{0}\right) \\
\left(-\operatorname{div}\left(\gamma_{1}^{\prime}\left(k_{1}^{*}\right) m_{1} \nabla k_{1}^{*}\right), 0\right) \\
\left(-\operatorname{div}\left(\alpha_{2}^{\prime}\left(k_{2}^{*}\right) m_{2} \nabla \boldsymbol{u}_{2}^{*}\right), \mathbf{0}\right) \\
\left(-\operatorname{div}\left(\gamma_{1}^{\prime}\left(k_{2}^{*}\right) m_{2} \nabla k_{2}^{*}\right), 0\right)
\end{array}\right)
$$

A similar formula would hold for the discrete problem

$$
\begin{aligned}
D \mathcal{T}_{\delta}\left(k_{1}^{*}, k_{2}^{*}\right) \mathcal{G}\left(U_{1}^{*}, U_{2}^{*}\right)+\mathcal{T}_{\delta}\left(k_{1}^{*}, k_{2}^{*}\right) & D \mathcal{G}\left(U_{1}^{*}, U_{2}^{*}\right) \\
& =\mathcal{T}_{\delta}\left(k_{1}^{*}, k_{2}^{*}\right)\left(D \mathcal{G}\left(U_{1}^{*}, U_{2}^{*}\right)+D \mathcal{H}_{\delta}\left(U_{1}^{*}, U_{2}^{*}\right)\right)
\end{aligned}
$$

where the duality product of the first part of the first and third components with a $\boldsymbol{v}_{i \delta}$ in $V_{i \delta}$, respectively, of the second and fourth components with a $\ell_{i \delta}$ in $Y_{\delta}$, are given by the formulas

$$
\left(\alpha_{i}^{\prime}\left(k_{i}^{*}\right) m_{i \delta} \nabla \boldsymbol{u}_{i}^{*}, \nabla \boldsymbol{v}_{i \delta}\right)_{i \delta}, \quad\left(\gamma_{i}^{\prime}\left(k_{i}^{*}\right) m_{i \delta} \nabla k_{i}^{*}, \nabla \ell_{i \delta}\right)_{i \delta} .
$$


(Here, the differential operator is applied to a $W_{\delta}=\left(W_{1 \delta}, W_{2 \delta}\right)$ in $\mathcal{X}_{\delta}$, with each $W_{i \delta}$ equal to $\left(\boldsymbol{w}_{i \delta}, m_{i \delta}\right)$.)

From now on, we fix $\varepsilon$ such that $2 \varepsilon<s^{*}-\frac{d}{2}$.

LEMma 5.1. If Hypothesis 2.2 is satisfied, the following property holds:

$$
\lim _{K_{1} \rightarrow+\infty, K_{2} \rightarrow+\infty}\left\|\mathcal{O}_{1}\right\|_{\mathrm{L}}\left(\mathcal{X}_{\delta}, \mathcal{X}_{\delta}\right)=0 .
$$

Proof. Due to formulas (5.6) and (5.7), we observe that

$$
\begin{aligned}
\left\|\mathcal{O}_{1}\right\|_{\mathrm{L}}\left(\mathcal{X}_{\delta}, \mathcal{X}_{\delta}\right) \leq \|\left(\mathcal{T}-\mathcal{T}_{\delta}\right) & \left(k_{1}^{*}, k_{2}^{*}\right)\left(D \mathcal{G}\left(U_{1}^{*}, U_{2}^{*}\right)+D \mathcal{H}\left(U_{1}^{*}, U_{2}^{*}\right)\right) \|_{\mathrm{L}}\left(\mathcal{X}_{\delta}, \mathcal{X}_{\delta}\right) \\
& +\left\|\mathcal{T}_{\delta}\left(k_{1}^{*}, k_{2}^{*}\right)\left(D \mathcal{H}\left(U_{1}^{*}, U_{2}^{*}\right)-D \mathcal{H}_{\delta}\left(U_{1 \delta^{\prime}}^{*}, U_{2 \delta^{\prime}}^{*}\right)\right)\right\|_{\mathrm{L}}\left(\mathcal{X}_{\delta}, \mathcal{X}_{\delta}\right) .
\end{aligned}
$$

(1) To bound the first term, we note that, when $\left(W_{1 \delta}, W_{2 \delta}\right)$ runs through the unit sphere of $\mathcal{X}_{\delta}$, the quantities

$$
D \boldsymbol{\lambda}_{i}\left(\boldsymbol{u}_{1}^{*}, \boldsymbol{u}_{2}^{*}\right) \cdot\left(\boldsymbol{w}_{1 \delta}, \boldsymbol{w}_{2 \delta}\right), \quad D g_{i}\left(k_{i}^{*}, \boldsymbol{u}_{i}^{*}\right) \cdot\left(m_{i \delta}, \boldsymbol{w}_{i \delta}\right), \quad \text { and } \quad D \lambda\left(\boldsymbol{u}_{1}^{*}, \boldsymbol{u}_{2}^{*}\right) \cdot\left(\boldsymbol{w}_{1 \delta}, \boldsymbol{w}_{2 \delta}\right)
$$

belong to a bounded set in $L^{2}\left(\Omega_{i}\right)^{d}, H^{-1}\left(\Omega_{i}\right)$, and $H_{00}^{\frac{1}{2}}(\Gamma)$, respectively. Then it can be checked that $\mathcal{S}_{i}\left(k_{i}^{*}\right)\left(\mathbf{0}, D \boldsymbol{\lambda}_{i}\left(\boldsymbol{u}_{1}^{*}, \boldsymbol{u}_{2}^{*}\right) \cdot\left(\boldsymbol{w}_{1 \delta}, \boldsymbol{w}_{2 \delta}\right)\right)$ remains inside a bounded set of $H^{s}\left(\Omega_{i}\right)^{d}$ for some $s>1$. Thanks to (4.18), (4.19), and the analogue of (4.11), this yields the uniform convergence of $\left(\mathcal{S}_{i}-\mathcal{S}_{i \delta}\right)\left(k_{i}^{*}\right)\left(\mathbf{0}, D \boldsymbol{\lambda}_{i}\left(\boldsymbol{u}_{1}^{*}, \boldsymbol{u}_{2}^{*}\right) \cdot\left(\boldsymbol{w}_{1 \delta}, \boldsymbol{w}_{2 \delta}\right)\right)$. The convergence of the other terms, say

$$
\left(\mathcal{L}_{i}-\mathcal{L}_{i \delta}\right)\left(k_{i}^{*}\right)\left(D g_{i}\left(k_{i}^{*}, \boldsymbol{u}_{i}^{*}\right) \cdot\left(m_{i \delta}, \boldsymbol{w}_{i \delta}\right), D \lambda\left(\boldsymbol{u}_{1}^{*}, \boldsymbol{u}_{2}^{*}\right) \cdot\left(\boldsymbol{w}_{1 \delta}, \boldsymbol{w}_{2 \delta}\right)\right),
$$

follows from (4.13) together with the fact that both

$$
\mathcal{L}_{i}\left(D g_{i}\left(k_{i}^{*}, \boldsymbol{u}_{i}^{*}\right) \cdot\left(m_{i}, \boldsymbol{w}_{i}\right), D \lambda\left(\boldsymbol{u}_{1}^{*}, \boldsymbol{u}_{2}^{*}\right) \cdot\left(\boldsymbol{w}_{1}, \boldsymbol{w}_{2}\right)\right)
$$

and its discrete analogue

$$
\mathcal{L}_{i \delta}\left(k_{i}^{*}\right)\left(D g_{i}\left(k_{i}^{*}, \boldsymbol{u}_{i}^{*}\right) \cdot\left(m_{i}, \boldsymbol{w}_{i}\right), D \lambda\left(\boldsymbol{u}_{1}^{*}, \boldsymbol{u}_{2}^{*}\right) \cdot\left(\boldsymbol{w}_{1}, \boldsymbol{w}_{2}\right)\right)
$$

are bounded in $H^{1}\left(\Omega_{i}\right)$; see (4.7). So combining these facts yields

$$
\lim _{K_{1} \rightarrow+\infty, K_{2} \rightarrow+\infty}\left\|\left(\mathcal{T}-\mathcal{T}_{\delta}\right)\left(k_{1}^{*}, k_{2}^{*}\right) D \mathcal{G}\left(U_{1}^{*}, U_{2}^{*}\right)\right\|_{\mathrm{L}(\mathcal{X}, \mathcal{X})}=0
$$

(2) Similarly, we observe that, when $\left(W_{1 \delta}, W_{2 \delta}\right)$ runs through the unit sphere of $\mathcal{X}_{\delta}$, the quantities $\alpha_{i}^{\prime}\left(k_{i}^{*}\right) m_{i \delta} \nabla \boldsymbol{u}_{i}^{*}$ and $\gamma_{i}^{\prime}\left(k_{i}^{*}\right) m_{i \delta} \nabla k_{i}^{*}$ belong to a bounded set of $H^{s}\left(\Omega_{i}\right)^{d^{2}}$ and $H^{s}\left(\Omega_{i}\right)^{d}$, for some $s>0$, so that their divergence belongs to $H^{s-1}\left(\Omega_{i}\right)^{d}$ and $H^{s-1}\left(\Omega_{i}\right)$, respectively. This implies

$$
\lim _{K_{1} \rightarrow+\infty, K_{2} \rightarrow+\infty}\left\|\left(\mathcal{T}-\mathcal{T}_{\delta}\right)\left(k_{1}^{*}, k_{2}^{*}\right) D \mathcal{H}\left(U_{1}^{*}, U_{2}^{*}\right)\right\|_{\mathrm{L}(\mathcal{X}, \mathcal{X})}=0 .
$$

(3) To prove the convergence of the last term, we observe from (4.8) that it suffices to prove, for any $m_{i \delta}$ in the intersection of $\mathbb{P}_{K_{i}, N_{i}}\left(\Omega_{i}\right)$ with the unit sphere of $H^{1-\varepsilon}\left(\Omega_{i}\right)$, the convergence of

$$
\sup _{\ell_{i \delta} \in Y_{i \delta}} \frac{\int_{\Omega_{i}} \gamma_{i}^{\prime}\left(k_{i}^{*}\right) m_{i \delta} \nabla k_{i}^{*} \cdot \nabla \ell_{i \delta} d \boldsymbol{x}-\left(\gamma_{i}^{\prime}\left(k_{i}^{*}\right) m_{i \delta} \nabla k_{i \delta^{\prime}}^{*}, \nabla \ell_{i \delta}\right)_{i \delta}}{\left\|\ell_{i \delta}\right\|_{H^{1}\left(\Omega_{i}\right)}} .
$$


Handling the other terms is similar. Denoting by $\delta^{\prime \prime}$ the 4 -tuple $\left(K_{1}^{\prime \prime}, N_{1}^{\prime \prime}, K_{2}^{\prime \prime}, N_{2}^{\prime \prime}\right)$ with each $K_{i}^{\prime \prime}$ equal to the integral part of $\frac{K_{i}-1}{4}$ and each $N_{i}^{\prime \prime}$ equal to the integral part of $\frac{N_{i}-1}{4}$, we derive from the exactness of the quadrature formula for any $\tilde{\gamma}_{i \delta^{\prime \prime}}$ and $m_{i \delta^{\prime \prime}}$ in $\mathbb{P}_{K_{i}^{\prime \prime}, N_{i}^{\prime \prime}}\left(\Omega_{i}\right)$,

$$
\int_{\Omega_{i}} \gamma_{i \delta^{\prime \prime}} m_{i \delta^{\prime \prime}} \nabla k_{i \delta^{\prime}}^{*} \cdot \nabla \ell_{i \delta} d \boldsymbol{x}=\left(\gamma_{i \delta^{\prime \prime}} m_{i \delta^{\prime \prime}} \nabla k_{i \delta^{\prime}}^{*}, \nabla \ell_{i \delta}\right)_{i \delta}
$$

By adding and subtracting this line, we prove that the previous quantity is bounded by the sum of the terms

$$
\begin{aligned}
& \left\|\gamma_{i}^{\prime}\left(k_{i}^{*}\right)\right\|_{L^{\infty}\left(\Omega_{i}\right)}\left\|m_{i \delta} \nabla\left(k_{i}^{*}-k_{i \delta^{\prime}}^{*}\right)\right\|_{L^{2}\left(\Omega_{i}\right)^{d}}, \\
& \left\|\gamma_{i}^{\prime}\left(k_{i}^{*}\right)\right\|_{L^{\infty}\left(\Omega_{i}\right)}\left\|\left(m_{i \delta}-m_{i \delta^{\prime \prime}}\right) \nabla k_{i \delta^{\prime}}^{*}\right\|_{L^{2}\left(\Omega_{i}\right)^{d}}, \\
& \left\|\gamma_{i}^{\prime}\left(k_{i}^{*}\right)-\tilde{\gamma}_{i \delta^{\prime \prime}}\right\|_{L^{\infty}\left(\Omega_{i}\right)}\left\|m_{i \delta^{\prime \prime}} \nabla k_{i \delta^{\prime}}^{*}\right\|_{L^{2}\left(\Omega_{i}\right)^{d}}, \\
& \left\|\gamma_{i}^{\prime}\left(k_{i}^{*}\right)\right\|_{L^{\infty}\left(\Omega_{i}\right)}\left\|\mathcal{I}_{i \delta}\left(\left(m_{i \delta}-m_{i \delta^{\prime \prime}}\right) \nabla k_{i \delta^{\prime}}^{*}\right)\right\|_{L^{2}\left(\Omega_{i}\right)^{d}}, \\
& \left\|\gamma_{i}^{\prime}\left(k_{i \delta}^{*}\right)-\tilde{\gamma}_{i \delta^{\prime \prime}}\right\|_{L^{\infty}\left(\Omega_{i}\right)}\left\|\mathcal{I}_{i \delta}\left(m_{i \delta^{\prime \prime}} \nabla k_{i \delta^{\prime}}^{*}\right)\right\|_{L^{2}\left(\Omega_{i}\right)^{d}} .
\end{aligned}
$$

Thanks to the choice of $\varepsilon$, the product of two functions is continuous from $H^{1-\varepsilon}\left(\Omega_{i}\right) \times H^{s^{*}-1-\varepsilon}\left(\Omega_{i}\right)$ into $L^{2}\left(\Omega_{i}\right)$ so that the uniform convergence of the first term follows from (5.2). Again, thanks to the choice of $\varepsilon$, the product of two functions is continuous from $H^{1-2 \varepsilon}\left(\Omega_{i}\right) \times H^{s^{*}-1}\left(\Omega_{i}\right)$ into $L^{2}\left(\Omega_{i}\right)$ so that the uniform convergence of the second term is obtained by taking $m_{i \delta^{\prime \prime}}$ equal to the projection of $m_{i \delta}$ onto $\mathbb{P}_{K_{i}^{\prime \prime}, N_{i}^{\prime \prime}}\left(\Omega_{i}\right)$ for the scalar product of $H^{1-\varepsilon}\left(\Omega_{i}\right)$ and using the approximation properties of this projection operator; see [7, Thm. 7.4]. Similarly, the convergence of the third term follows from (4.11). The convergence of the last two terms is derived by similar arguments combined with the stability on the operator $\mathcal{I}_{i \delta}$ on polynomials; see [7, Form. (13.28)].

So the proof is complete.

LEMma 5.2. If Hypothesis 2.2 is satisfied, the following property holds:

$$
\lim _{K_{1} \rightarrow+\infty, K_{2} \rightarrow+\infty}\left\|\mathcal{O}_{2}\right\|_{\mathrm{I}}\left(\mathcal{X}_{\delta}, \mathcal{X}_{\delta}\right)=0 .
$$

Proof. From (5.7), we have

$\left\|\mathcal{O}_{2}\right\|_{\mathrm{L}}\left(\mathcal{X}_{\delta}, \mathcal{X}_{\delta}\right)=\left\|\left(\mathcal{T}_{\delta}\left(k_{1}^{*}, k_{2}^{*}\right)-\mathcal{T}_{\delta}\left(k_{1 \delta^{\prime}}^{*}, k_{2 \delta^{\prime}}^{*}\right)\right)\left(D \mathcal{G}\left(U_{1}^{*}, U_{2}^{*}\right)+D \mathcal{H}_{\delta}\left(U_{1 \delta^{\prime}}^{*}, U_{2 \delta^{\prime}}^{*}\right)\right)\right\|_{\mathrm{L}}\left(\mathcal{X}_{\delta}, \mathcal{X}_{\delta}\right)$.

When $\left(W_{1 \delta}, W_{2 \delta}\right)$ runs through the unit sphere of $\mathcal{X}_{\delta}$, the quantity

$$
\mathcal{T}_{\delta}\left(k_{1}^{*}, k_{2}^{*}\right)\left(D \mathcal{G}\left(U_{1}^{*}, U_{2}^{*}\right)+D \mathcal{H}_{\delta}\left(U_{1}^{*}, U_{2 \delta^{\prime}}^{*}\right)\right)
$$

remains bounded in $\mathcal{X}$ so that the desired convergence result follows from (4.14) and (4.20), combined with (5.2) and the embedding of $H^{s}\left(\Omega_{i}\right)$ into $L^{\infty}\left(\Omega_{i}\right)$ for all $s>\frac{d}{2}$.

LEMma 5.3. If Hypothesis 2.2 is satisfied, the following property holds:

$$
\lim _{K_{1} \rightarrow+\infty, K_{2} \rightarrow+\infty}\left\|\mathcal{O}_{3}\right\|_{\mathrm{L}}\left(\mathcal{X}_{\delta}, \mathcal{X}_{\delta}\right)=0 .
$$

Proof. Here, the convergence of each term is a straightforward consequence of (5.2).

Lemma 5.4. If Hypothesis 2.2 is satisfied and if the data $\boldsymbol{f}_{i}, 1 \leq i \leq 2$, belong to $H^{\sigma}\left(\Omega_{i}\right)^{d}$ for some $\sigma>\frac{d}{2}$, the following property holds:

$$
\lim _{K_{1} \rightarrow+\infty, K_{2} \rightarrow+\infty}\left\|\mathcal{O}_{4}\right\|_{\perp}\left(\mathcal{X}_{\delta}, \mathcal{X}_{\delta}\right)=0 .
$$


Proof. Thanks to (4.8) and its analogue for the Stokes problem, the convergence of the term

$$
\left\|\mathcal{T}_{\delta}\left(k_{1 \delta}^{*}, k_{2 \delta}^{*}\right)\left(D \mathcal{G}\left(U_{1 \delta^{\prime}}^{*}, U_{2 \delta^{\prime}}^{*}\right)-D \mathcal{G}_{\delta}\left(U_{1 \delta^{\prime}}^{*}, U_{2 \delta^{\prime}}^{*}\right)\right)\right\|_{\mathrm{L}\left(\mathcal{X}_{\delta}, \mathcal{X}_{\delta}\right)}
$$

is a consequence of the convergence of the terms

$$
\begin{aligned}
& \sup _{\boldsymbol{w}_{i \delta} \in X_{i \delta}} \frac{\int_{\Omega_{i}} \alpha_{i}\left(k_{i \delta}^{*}\right) \nabla \boldsymbol{u}_{i \delta}^{*} \cdot \nabla \boldsymbol{w}_{i \delta} d \boldsymbol{x}-\left(\alpha_{i}\left(k_{i \delta}^{*}\right) \nabla \boldsymbol{u}_{i \delta}^{*}, \nabla \boldsymbol{w}_{i \delta}\right)_{i \delta}}{\left\|\boldsymbol{w}_{i \delta}\right\|_{H^{1}\left(\Omega_{i}\right)^{d}}}, \\
& \sup _{m_{i \delta} \in Y_{i \delta}} \frac{\int_{\Omega_{i}} \alpha_{i}^{\prime}\left(k_{i \delta}^{*}\right) m_{i \delta} \nabla \boldsymbol{u}_{i \delta}^{*} \cdot \nabla \boldsymbol{u}_{i \delta}^{*} d \boldsymbol{x}-\left(\alpha_{i}^{\prime}\left(k_{i \delta}^{*}\right) m_{i \delta} \nabla \boldsymbol{u}_{i \delta}^{*}, \nabla \boldsymbol{u}_{i \delta}^{*}\right)_{i \delta}}{\left\|m_{i \delta}\right\|_{H^{1-\varepsilon}\left(\Omega_{i}\right)}}
\end{aligned}
$$

and of their analogues for the Stokes problem. As in the end of the proof of Lemma 4.1, this is obtained by adding and subtracting appropriate terms of lower degree.

Similar arguments yield the convergence of $\mathcal{G}_{\delta}\left(U_{1 \delta^{\prime}}^{*}, U_{2 \delta^{\prime}}^{*}\right)$ toward $\mathcal{G}\left(U_{1 \delta^{\prime}}^{*}, U_{2 \delta^{\prime}}^{*}\right)$, whence the convergence of the second term in $\mathcal{O}_{4}$.

Combining the results of Lemmas 5.1 to 5.4 leads to the following result.

Proposition 5.5. If Hypotheses 2.2 and 2.4 are satisfied and if the data $\boldsymbol{f}_{i}$, $1 \leq i \leq 2$, belong to $H^{\sigma}\left(\Omega_{i}\right)^{d}$ for some $\sigma>\frac{d}{2}$, there exists a constant $K$ such that, for $K_{1} \geq K$ and $K_{2} \geq K$, the operator

$$
\operatorname{Id}+D \mathcal{T}_{\delta}\left(k_{1 \delta^{\prime}}^{*}, k_{2 \delta^{\prime}}^{*}\right) \mathcal{G}_{\delta}\left(U_{1 \delta^{\prime}}^{*}, U_{2 \delta^{\prime}}^{*}\right)+\mathcal{T}_{\delta}\left(k_{1 \delta^{\prime}}^{*}, k_{2 \delta^{\prime}}^{*}\right) D \mathcal{G}_{\delta}\left(U_{1 \delta^{\prime}}^{*}, U_{2 \delta^{\prime}}^{*}\right)
$$

is an isomorphism of $\mathcal{X}_{\delta}$. Moreover, the norm of its inverse is bounded by a constant $\gamma$ independent of $K_{1}$ and $K_{2}$.

The following proposition states a Lipschitz property for the discrete operator. Since its proof is simpler than for the previous result, we only sketch it.

Proposition 5.6. The following property holds for all nonnegative real numbers $\alpha$ and for any $\left(Z_{1 \delta}, Z_{2 \delta}\right)$ in $\mathcal{X}_{\delta}$ which satisfies $\left\|\left(Z_{1 \delta}, Z_{2 \delta}\right)-\left(U_{1 \delta^{\prime}}^{*}, U_{2 \delta^{\prime}}^{*}\right)\right\|_{\mathcal{X}} \leq \alpha$, with $Z_{i \delta}=\left(\boldsymbol{z}_{i \delta}, r_{i \delta}\right)$ :

$$
\begin{aligned}
& \| D \mathcal{T}_{\delta}\left(k_{1 \delta^{\prime}}^{*}, k_{2 \delta^{\prime}}^{*}\right) \mathcal{G}_{\delta}\left(U_{1 \delta^{\prime}}^{*}, U_{2 \delta^{\prime}}^{*}\right)+\mathcal{T}_{\delta}\left(k_{1 \delta^{\prime}}^{*}, k_{2 \delta^{\prime}}^{*}\right) D \mathcal{G}_{\delta}\left(U_{1 \delta^{\prime}}^{*}, U_{2 \delta^{\prime}}^{*}\right) \\
& \quad-D \mathcal{T}_{\delta}\left(r_{1 \delta}, r_{2 \delta}\right) \mathcal{G}_{\delta}\left(Z_{1 \delta}, Z_{2 \delta}\right)-\mathcal{T}_{\delta}\left(r_{1 \delta}, r_{2 \delta}\right) D \mathcal{G}_{\delta}\left(Z_{1 \delta}, Z_{2 \delta}\right) \|_{\mathrm{L}}\left(\mathcal{X}_{\delta}, \mathcal{X}_{\delta}\right) \leq c \kappa_{\delta} \alpha
\end{aligned}
$$

where according to the dimension $d$ the constant $\kappa_{\delta}$ is given by

$$
\kappa_{\delta}=K_{i}^{2(d-2)+2 \varepsilon}\left(\log K_{i}\right)^{\frac{3-d}{2}} .
$$

Proof. We must bound the four terms

$$
\begin{array}{r}
\left\|\mathcal{T}_{\delta}\left(r_{1 \delta}, r_{2 \delta}\right)\left(D \mathcal{G}_{\delta}\left(U_{1 \delta^{\prime}}^{*}, U_{2 \delta^{\prime}}^{*}\right)-D \mathcal{G}_{\delta}\left(Z_{1 \delta}, Z_{2 \delta}\right)\right)\right\|_{\mathrm{L}}\left(\mathcal{X}_{\delta}, \mathcal{X}_{\delta}\right) \\
\left\|\left(\mathcal{T}_{\delta}\left(k_{1 \delta^{\prime}}^{*}, r_{2 \delta^{\prime}}^{*}\right)-\mathcal{T}_{\delta}\left(r_{1 \delta}, r_{2 \delta}\right)\right) D \mathcal{G}_{\delta}\left(U_{1 \delta^{\prime}}^{*}, U_{2 \delta^{\prime}}^{*}\right)\right\|_{\mathrm{L}\left(\mathcal{X}_{\delta}, \mathcal{X}_{\delta}\right)}, \\
\left\|\mathcal{T}_{\delta}\left(r_{1 \delta}, r_{2 \delta}\right)\left(D \mathcal{H}_{\delta}\left(U_{1 \delta^{\prime}}^{*}, U_{2 \delta^{\prime}}^{*}\right)-D \mathcal{H}_{\delta}\left(Z_{1 \delta^{\prime}}, Z_{2 \delta^{\prime}}\right)\right)\right\|_{\mathrm{L}}\left(\mathcal{X}_{\delta}, \mathcal{X}_{\delta}\right) \\
\quad\left\|\left(\mathcal{T}_{\delta}\left(k_{1 \delta^{\prime}}^{*}, r_{2 \delta^{\prime}}^{*}\right)-\mathcal{T}_{\delta}\left(r_{1 \delta}, r_{2 \delta}\right)\right) D \mathcal{H}_{\delta}\left(U_{1 \delta^{\prime}}^{*}, U_{2 \delta^{\prime}}^{*}\right)\right\|_{\mathrm{L}\left(\mathcal{X}_{\delta}, \mathcal{X}_{\delta}\right)} .
\end{array}
$$

For instance, in the first term, we must bound the quantity, for $m_{i \delta}$ running through the unit ball of $Y_{i \delta}$,

$$
\sum_{\ell_{i \delta} \in Y_{i \delta}} \frac{\left(\alpha_{i}^{\prime}\left(k_{i \delta^{\prime}}^{*}\right) m_{i \delta}\left|\nabla\left(\boldsymbol{u}_{i \delta^{\prime}}-z_{i \delta}\right)\right|^{2}, \ell_{i \delta}\right)_{i \delta}}{\left\|\ell_{i \delta}\right\|_{H^{1}\left(\Omega_{i}\right)}} .
$$


Applying the inverse inequalities

$$
\forall \varphi_{i \delta} \in \mathbb{P}_{K_{i}, N_{i}}\left(\Omega_{i}\right), \quad\left\|\varphi_{i \delta}\right\|_{L^{\infty}\left(\Omega_{i}\right)} \leq\left\{\begin{array}{l}
c K_{i}^{d-2+2 \varepsilon}\left\|\varphi_{i \delta}\right\|_{H^{1-\varepsilon}\left(\Omega_{i}\right)}, \\
c K_{i}^{d-2}\left(\log K_{i}\right)^{\frac{3-d}{2}}\left\|\varphi_{i \delta}\right\|_{H^{1}\left(\Omega_{i}\right)},
\end{array}\right.
$$

we have to bound the term, for all $m_{i \delta}$ in $\mathbb{P}_{K_{i}, N_{i}}\left(\Omega_{i}\right)$,

$$
K_{i}^{2(d-2)+2 \varepsilon}\left(\log K_{i}\right)^{\frac{3-d}{2}}\left\|\nabla\left(\boldsymbol{u}_{i \delta^{\prime}}^{*}-\boldsymbol{z}_{i \delta}\right)\right\|_{L^{2}\left(\Omega_{i}\right)}\left\|\nabla\left(\boldsymbol{u}_{i \delta^{\prime}}^{*}+\boldsymbol{z}_{i \delta}\right)\right\|_{L^{2}\left(\Omega_{i}\right)} .
$$

This yields the value of $\kappa_{\delta}$. The other terms are simpler; they can be evaluated by similar arguments.

Finally, we must evaluate the quantity

$$
\varepsilon_{\delta}=\left\|\left(\begin{array}{c}
U_{1 \delta^{\prime}}^{*} \\
U_{2 \delta^{\prime}}^{*}
\end{array}\right)+\mathcal{T}_{\delta}\left(k_{1 \delta^{\prime}}^{*}, k_{2 \delta^{\prime}}^{*}\right) \mathcal{G}_{\delta}\left(U_{1 \delta^{\prime}}^{*}, U_{2 \delta^{\prime}}^{*}\right)\right\|_{\mathcal{X}} .
$$

Proposition 5.7. If Hypothesis 2.2 is satisfied and if the data $\boldsymbol{f}_{i}, 1 \leq i \leq 2$, belong to $H^{\sigma}\left(\Omega_{i}\right)^{d}$ for some $\sigma>\frac{d}{2}$, the following estimate holds for a constant $C$ only depending on the norm of $\left(U_{1}^{*}, U_{2}^{*}\right)$ in $\prod_{i=1}^{2}\left(H^{s^{*}}\left(\Omega_{i}\right)^{d} \times H^{s^{*}}\left(\Omega_{i}\right)\right)$ :

$$
\varepsilon_{\delta} \leq C\left(\inf \left\{K_{1}, K_{2}\right\}\right)^{1-s^{*}} .
$$

Proof. By using formulation (2.18), we observe that

$$
\begin{gathered}
\varepsilon_{\delta} \leq\left\|U_{1}^{*}-U_{1 \delta}^{*}\right\|_{\mathcal{X}_{1}}+\left\|U_{2}^{*}-U_{2 \delta}^{*}\right\|_{\mathcal{X}_{2}} \\
+\left\|\left(\mathcal{T}-\mathcal{T}_{\delta}\right)\left(k_{1}^{*}, k_{2}^{*}\right) \mathcal{G}\left(U_{1}^{*}, U_{2}^{*}\right)\right\|_{\mathcal{X}}+\left\|\left(\mathcal{T}_{\delta}\left(k_{1}^{*}, k_{2}^{*}\right)-\mathcal{T}_{\delta}\left(k_{1 \delta^{\prime}}^{*}, k_{2 \delta^{\prime}}^{*}\right)\right) \mathcal{G}\left(U_{1}^{*}, U_{2}^{*}\right)\right\|_{\mathcal{X}} \\
+\left\|\mathcal{T}_{\delta}\left(k_{1 \delta^{\prime}}^{*}, k_{2 \delta^{\prime}}^{*}\right)\left(\mathcal{G}\left(U_{1}^{*}, U_{2}^{*}\right)-\mathcal{G}\left(U_{1 \delta^{\prime}}^{*}, U_{2 \delta^{\prime}}^{*}\right)\right)\right\|_{\mathcal{X}} \\
+\left\|\mathcal{T}_{\delta}\left(k_{1 \delta^{\prime}}^{*}, k_{2 \delta^{\prime}}^{*}\right)\left(\mathcal{G}\left(U_{1 \delta^{\prime}}^{*}, U_{2 \delta^{\prime}}^{*}\right)-\mathcal{G}_{\delta}\left(U_{1 \delta^{\prime}}^{*}, U_{2 \delta^{\prime}}^{*}\right)\right)\right\|_{\mathcal{X}} .
\end{gathered}
$$

The bound for the first two terms in the right-hand side comes from (5.2), and the bound for the third term is derived from (4.9) and its analogue for the Stokes problem together with the regularity Hypothesis 2.2. The fourth term is easily bounded from (4.14) and (4.20), while estimating the fifth one relies on (4.7) and (4.17), combined with (5.2). Finally, estimating the last term also relies on (4.7) and (4.17) together with the introduction of approximations of $\alpha_{i}\left(k_{i}^{*}\right)$ and $\gamma_{i}\left(k_{i}^{*}\right)$ in $\mathbb{P}_{K_{i}^{\prime}, N_{i}^{\prime}}\left(\Omega_{i}\right)$; see (4.12).

We are now in a position to apply the Brezzi-Rappaz-Raviart theorem [10] (see also [15, Chap. IV, Thm. 3.1]).

THEOREM 5.8. If Hypotheses 2.2 and 2.4 are satisfied with $s^{*}>2(d-2)+1$ and if the data $\boldsymbol{f}_{i}, i=1,2$, belong to $H^{\sigma}\left(\Omega_{i}\right)^{d}$ for some $\sigma>\sup \left\{\frac{d}{2}, s^{*}-1\right\}$, there exist an integer $K_{0}$ and a constant $\lambda$ such that, for $K_{1} \geq K_{0}$ and $K_{2} \geq K_{0}$, problem (3.4)-(3.7) has a unique solution $\left(U_{1 \delta}, U_{2 \delta}\right)$, with each $U_{i \delta}$ equal to $\left(\boldsymbol{u}_{i \delta}, k_{i \delta}\right)$, in the neighborhood $\mathrm{U}$ of $U=\left(U_{1}^{*}, U_{2}^{*}\right)$ defined as follows:

$$
\mathrm{U}=\left\{Z_{\delta}=\left(Z_{1 \delta}, Z_{2 \delta}\right) \in \mathcal{X}_{\delta} ;\left\|U-Z_{\delta}\right\|_{\mathcal{X}} \leq \lambda \kappa_{\delta}^{-1}\right\} .
$$

Moreover, the following error estimates hold for $i=1$ and 2 :

$$
\left\|\boldsymbol{u}_{i}^{*}-\boldsymbol{u}_{i \delta}\right\|_{H^{1}\left(\Omega_{i}\right)^{d}}+\left\|k_{i}^{*}-k_{i \delta}\right\|_{L^{2}\left(\Omega_{i}\right)} \leq C_{0}\left(\inf \left\{K_{1}, K_{2}\right\}\right)^{1-s^{*}}
$$

for a constant $C_{0}$ depending only on the norms of $\left(U_{1}^{*}, U_{2}^{*}\right)$ in $\prod_{i=1}^{2}\left(H^{s^{*}}\left(\Omega_{i}\right)^{d} \times\right.$ $\left.H^{s^{*}}\left(\Omega_{i}\right)\right)$ and of $\left(\boldsymbol{f}_{1}, \boldsymbol{f}_{2}\right)$ in $\prod_{i=1}^{2} H^{\sigma}\left(\Omega_{i}\right)^{d}$. 
We conclude with an estimate on the pressure, which is easily derived from the inf-sup condition established in Lemma 3.4.

COROLlary 5.9. If the assumptions of Theorem 5.8 are satisfied, for the solution $\left(U_{1 \delta}, U_{2 \delta}\right)$ exhibited in Theorem 5.8 , there exists a unique pair $\left(p_{1 \delta}, p_{2 \delta}\right)$ in $M_{1 \delta}^{m} \times M_{2 \delta}^{m}$ such that $\left(\tilde{U}_{1 \delta}, \tilde{U}_{2 \delta}\right)$, with each $\tilde{U}_{i \delta}$ equal to $\left(\boldsymbol{u}_{i \delta}, p_{i \delta}, k_{i \delta}\right)$, is a solution of problem (3.4)-(3.5). Moreover, the following error estimates hold for $i=1$ and 2 :

$$
\left\|p_{i}^{*}-p_{i \delta}\right\|_{L^{2}\left(\Omega_{i}\right)} \leq C_{0}^{\prime}\left(\beta_{i \delta}^{m}\right)^{-1}\left(\inf \left\{K_{1}, K_{2}\right\}\right)^{1-s^{*}}
$$

for the constants $\beta_{i \delta}^{m}$ evaluated in (3.9) and a constant $C_{0}^{\prime}$ depending only on the norms of $\left(\tilde{U}_{1}^{*}, \tilde{U}_{2}^{*}\right)$ in $\prod_{i=1}^{2}\left(H^{s}\left(\Omega_{i}\right)^{d} \times H^{s-1}\left(\Omega_{i}\right) \times H^{s}\left(\Omega_{i}\right)\right)$ and of $\left(\boldsymbol{f}_{1}, \boldsymbol{f}_{2}\right)$ in $\prod_{i=1}^{2} H^{\sigma}\left(\Omega_{i}\right)^{d}$.

6. Conclusions and numerical algorithms. The regularity assumptions in Theorem 5.8 in the three-dimensional case are very unlikely; however, they seem unavoidable. This comes from the fact that the linearized problem (2.20) and (2.21) makes sense only for a smooth solution $U^{*}$. Nevertheless, this does not prevent the convergence of numerical experiments.

In contrast, the assumptions of Theorem 5.8 are fully reasonable in the twodimensional case and, if these assumptions hold, optimal error estimates are derived for the velocity, the kinetic energy, and the pressure for appropriate choices of the spaces $M_{i \delta}$. In this case, the maximal regularity $s^{*}$ would very likely coincide with the $s_{0} \simeq 1.5946$ introduced in section 2 so that the error would be smaller than $C_{0}\left(\inf \left\{K_{1}, K_{2}\right\}\right)^{-0.5946}$. Moreover, in the case of the rectangle, the explicit form of the singular functions associated with the Stokes operator with Dirichlet-Neumann boundary conditions is known [20]. As usual [6], this would lead to double the convergence order: for smooth enough data $\boldsymbol{f}_{i}, i=1$ and 2 , the error would be smaller than $C_{0}\left(\inf \left\{K_{1}, K_{2}\right\}\right)^{-1.1892}$.

The most standard choice of an operator $\Pi_{i \delta}^{\Gamma}$ satisfying (5.3) would be the orthogonal projection operator $\Pi_{K_{i}}^{\frac{1}{2}}$ from $H_{00}^{\frac{1}{2}}(\Gamma)$ onto $\mathbb{P}_{K_{i}}(\Gamma) \cap H_{00}^{\frac{1}{2}}(\Gamma)$; however, computing this operator is not easy. So we take $\Pi_{i \delta}^{\Gamma}$ equal to $\mathcal{I}_{i \delta}^{\Gamma} \Pi_{K_{i}}^{\frac{1}{2}}$. Indeed, estimate (5.3) is still satisfied by this operator and, in this case, the boundary conditions at the interface can be written in a very simple way:

$$
k_{i \delta}=\mathcal{I}_{i \delta}^{\Gamma}\left(\left|\boldsymbol{u}_{1 \delta}-\boldsymbol{u}_{2 \delta}\right|^{2}\right) \text { on } \Gamma \text {. }
$$

Note, moreover, that in the present situation there is no theoretical reason to choose $K_{1} \neq K_{2}$ and that, when $K_{1}$ and $K_{2}$ coincide, these conditions are still less expensive to enforce. However, for more complex geometries (for instance, if the $\Omega_{i}$ are convex quadrilaterals), different values of $K_{1}$ and $K_{2}$ can be needed since the regularity properties of the velocities in $\Omega_{1}$ and $\Omega_{2}$ are different.

Exactly the same arguments as for Theorem 5.8 prove [10] the convergence of Newton's algorithm for solving the nonlinear problem (3.4)-(3.5), when the initial guess $\left(U_{1 \delta}^{0}, U_{2 \delta}^{0}\right)$ belongs to the domain $\mathrm{U}$ introduced in (5.17); however, this method seems too expensive for the present problem. Instead of this, for an initial guess $\left(U_{1 \delta}^{0}, U_{2 \delta}^{0}\right)$, we propose to solve iteratively the following problem: if the pair $\left(\tilde{U}_{1 \delta}^{n}, \tilde{U}_{2 \delta}^{n}\right)$, with $\tilde{U}_{i \delta}^{n}=\left(\boldsymbol{u}_{i \delta}^{n}, p_{i \delta}^{n}, k_{i \delta}^{n}\right)$, is supposed to be known, then we solve the following problem: 
Find $\left(\boldsymbol{u}_{i \delta}^{n+1}, p_{i \delta}^{n+1}\right)$ in $X_{i \delta} \times M_{i \delta}$ such that

$$
\begin{aligned}
& \forall \boldsymbol{v}_{i \delta} \in X_{i \delta}, \\
& \quad a_{i \delta}\left(k_{i \delta}^{n} ; \boldsymbol{u}_{i \delta}^{n+1}, \boldsymbol{v}_{i \delta}\right)+b_{i \delta}\left(\boldsymbol{v}_{i \delta}, p_{i \delta}^{n+1}\right) \\
& +\left(\left|\boldsymbol{u}_{i \delta}^{n}-\boldsymbol{u}_{j \delta}^{n}\right|\left(\boldsymbol{u}_{i \delta}^{n+1}-\boldsymbol{u}_{j \delta}^{n+1}\right), \boldsymbol{v}_{i \delta}\right)_{i \delta}^{\Gamma}=\left(\boldsymbol{f}_{i}, \boldsymbol{v}_{i \delta}\right)_{i \delta}, \\
& \forall q_{i \delta} \in M_{i \delta}, \quad b_{i \delta}\left(\boldsymbol{u}_{i \delta}^{n+1}, q_{i \delta}\right)=0 ;
\end{aligned}
$$

Find $k_{i \delta}$ in $\mathbb{P}_{K_{i}, N_{i}}\left(\Omega_{i}\right)$, such that

$$
k_{i \delta}^{n+1}=0 \quad \text { on } \Gamma_{i} \quad \text { and } \quad k_{i \delta}^{n+1}=\mathcal{I}_{i \delta}^{\Gamma}\left(\left|\boldsymbol{u}_{1 \delta}^{n+1}-\boldsymbol{u}_{2 \delta}^{n+1}\right|^{2}\right) \quad \text { on } \Gamma \text {, }
$$

and

$$
\forall \varphi_{i \delta} \in Y_{i \delta}, \quad c_{i \delta}\left(k_{i \delta}^{n} ; k_{i \delta}^{n+1}, \varphi_{i \delta}\right)=\left(\alpha_{i}\left(k_{i \delta}^{n}\right)\left|\nabla \boldsymbol{u}_{i \delta}^{n+1}\right|^{2}, \varphi_{i \delta}\right)_{i \delta} .
$$

Clearly, these two linear problems are well-posed and the sequence $\left(\tilde{U}_{1 \delta}^{n}, \tilde{U}_{2 \delta}^{n}\right)_{n}$ converges. Numerical experiments to check the efficiency of the algorithm are under consideration.

\section{REFERENCES}

[1] Y. Achdou and O. Pironneau, Domain decomposition and wall laws, C. R. Acad. Sci. Paris Sér. I, 320 (1995), pp. 541-547.

[2] C. Bernardi, T. Chacón Rebollo, R. Lewandowski, and F. Murat, Existence d'une solution pour un modèle de deux fluides turbulents couplés, C. R. Acad. Sci. Paris Sér. I, 328 (1999), pp. 993-998.

[3] C. Bernardi, T. Chacón Rebollo, R. Lewandowski, and F. Murat, A model for two coupled turbulent fluids. Part I: Analysis of the system, in Nonlinear Partial Differential Equations and Their Applications, Collège de France Seminar, Vol. XIV, D. Cioranescu and J.-L. Lions, eds., Elsevier, Amsterdam, 2002, pp. 69-102.

[4] C. Bernardi, M. Dauge, and Y. Maday, Relèvement de traces préservant les polynômes, C. R. Acad. Sci. Paris Sér. I, 315 (1992), pp. 333-338.

[5] C. Bernardi, M. Dauge, And Y. Maday, Interpolation of nullspaces for polynomial approximation of divergence-free functions in a cube, in Boundary Value Problems and Integral Equations in Nonsmooth Domains, Lecture Notes in Pure and Appl. Math. 167, M. Costabel, M. Dauge, and S. Nicaise, eds., Dekker, New York, 1995, pp. 27-46.

[6] C. Bernardi and Y. Maday, Polynomial approximation of some singular functions, Appl. Anal., 42 (1991), pp. 1-32.

[7] C. Bernardi and Y. Maday, Spectral methods, in Handbook of Numerical Analysis V, P.G. Ciarlet and J.-L. Lions, eds., North-Holland, Amsterdam, 1997, pp. 209-485.

[8] C. Bernardi AND Y. Maday, Uniform inf-sup conditions for the spectral discretization of the Stokes problem, Math. Models Methods Appl. Sci., 9 (1999), pp. 395-414.

[9] J.M. Boland AND R.A. Nicolaides, Stability of finite elements under divergence constraints, SIAM J. Numer. Anal., 20 (1983), pp. 722-731.

[10] F. Brezzi, J. RAppaz, AND P.-A. RaViart, Finite dimensional approximation of nonlinear problems, Part I: Branches of nonsingular solutions, Numer. Math., 36 (1980), pp. 1-25.

[11] F. Brossier and R. Lewandowski, Impact of the variations of the mixing length in a first order turbulent closure system,, M2AN Modél. Math. Anal. Numér., 36 (2002), pp. 345372.

[12] J. Casado Diaz, T. Chacón Rebollo, M. Gómez Marmol, V. Girault, and F. Murat, Numerical approximation of a Laplace equation with $L_{1}$ data, submitted.

[13] C.M. Elliott and S. Larsson, A finite element model for the time-dependent Joule heating problem, Math. Comput., 64 (1995), pp. 1433-1453.

[14] D.A. French AND S.M.F. GARCIA, Finite element approximation of an evolution problem modeling shear band formation, Comput. Methods Appl. Mech. Engrg., 118 (1994), pp. 153161.

[15] V. Girault And P.-A. Raviart, Finite Element Methods for the Navier-Stokes Equations, Theory and Algorithms, Springer-Verlag, Berlin, 1986. 
[16] R. Lewandowski, Analyse mathématique et océanographie, Collection Recherches en Mathématiques Appliquées, Masson, Paris, 1997.

[17] J.-L. Lions And E. Magenes, Problèmes aux limites non homogènes et applications, Vol. 1, Dunod, Paris, 1968.

[18] J.-L. Lions, R. Temam, and S. Wang, Models for the coupled atmosphere and ocean, Comput. Mech. Adv., 1 (1993), pp. 1-120.

[19] Y. MADAY, Relèvement de traces polynomiales et interpolations hilbertiennes entre espaces de polynômes, C. R. Acad. Sci. Paris Sér. I, 309 (1989), pp. 463-468.

[20] M. ORLT AND A.-M. SÄNDIG, Regularity of viscous Navier-Stokes flows in nonsmooth domains, in Boundary Value Problems and Integral Equations in Nonsmooth Domains, Lecture Notes in Pure and Appl. Math. 167, M. Costabel, M. Dauge, and S. Nicaise, eds., Dekker, New York, 1995, pp. 185-201.

[21] C. PARÈs, Existence, uniqueness and regularity of solution of the equations of a turbulence model for incompressible fluids, Appl. Anal., 43 (1992), pp. 245-296.

[22] G. Sacchi Landriani and H. Vandeven, Polynomial approximation of divergence-free functions, Math. Comput., 52 (1989), pp. 103-130.

[23] G. Stampacchia, Équations elliptiques du second ordre à coefficients discontinus, Presses de l'Université de Montréal, Montréal, QB, Canada, 1966. 